\title{
Hardwood species classification with DWT based hybrid texture feature extraction techniques
}

\author{
ARVIND R YADAV $^{1, *}, \mathrm{R} S$ ANAND ${ }^{1}, \mathrm{M} \mathrm{L} \mathrm{DEWAL}^{1}$ and \\ SANGEETA GUPTA ${ }^{2}$ \\ ${ }^{1}$ Department of Electrical Engineering, Indian Institute of Technology Roorkee, \\ Roorkee, Uttarakhand 247667, India \\ ${ }^{2}$ Botany Division, Forest Research Institute Dehradun, Dehradun, \\ Uttarakhand 248006, India \\ e-mail: arvind.yadav.me@gmail.com; anandfee@iitr.ac.in; mohanfee@iitr.ac.in; \\ guptas@icfre.org
}

MS received 19 June 2014; revised 5 July 2015; accepted 2 August 2015

\begin{abstract}
In this work, discrete wavelet transform (DWT) based hybrid texture feature extraction techniques have been used to categorize the microscopic images of hardwood species into 75 different classes. Initially, the DWT has been employed to decompose the image up to 7 levels using Daubechies (db3) wavelet as decomposition filter. Further, first-order statistics (FOS) and four variants of local binary pattern (LBP) descriptors are used to acquire distinct features of these images at various levels. The linear support vector machine (SVM), radial basis function (RBF) kernel SVM and random forest classifiers have been employed for classification. The classification accuracy obtained with state-of-the-art and DWT based hybrid texture features using various classifiers are compared. The DWT based FOS-uniform local binary pattern (DWTFOSLBP ${ }^{\mathrm{u} 2}$ ) texture features at the $4^{\text {th }}$ level of image decomposition have produced best classification accuracy of $97.67 \pm 0.79 \%$ and $98.40 \pm 064 \%$ for grayscale and RGB images, respectively, using linear SVM classifier. Reduction in feature dataset by minimal redundancy maximal relevance (mRMR) feature selection method is achieved and the best classification accuracy of $99.00 \pm 0.79 \%$ and $99.20 \pm 0.42 \%$ have been obtained for DWT based FOS-LBP histogram Fourier features (DWTFOSLBP-HF) technique at the $5^{\text {th }}$ and $6^{\text {th }}$ levels of image decomposition for grayscale and RGB images, respectively, using linear SVM classifier. The DWTFOSLBP-HF features selected with mRMR method has also established superiority amongst the DWT based hybrid texture feature extraction techniques for randomly divided database into different proportions of training and test datasets.
\end{abstract}

Keywords. Hybrid texture features; linear SVM; RBF kernel SVM; local binary pattern; first-order statistics; discrete wavelet transform.

*For correspondence 


\section{Introduction}

Wood is a useful raw material for furniture, railway sleepers, tools, shipping and construction industry, etc. Researchers from different domains such as palaeontologists, archaeologists, forensic experts and art historians may be interested in the identification of wood (Wheeler \& Baas 1998). Wood is broadly classified as hardwood and softwood species. Softwood species are conifers and 90.00-95.00\% of their cells (called longitudinal tracheid) have simple cellular structure making it difficult to discriminate amongst themselves due to limited number of cell types. On the contrary, hardwood species (angiosperm) possess complex cellular structure and are clearly distinguishable among intra-species. For an illustration color images of few hardwood species are shown in figure 1. Anatomical characteristics like vessels, fibers, parenchyma's and rays play significant role in hardwood species identification (Hermanson \& Wiedenhoeft 2011). The unique cellular structure of each of the hardwood species varies widely among intra-species and serves as a signature for their identification (Bond 2002).

It is necessary to identify wood as its characteristics vary widely (Wang et al 2013). Accurate recognition of wood species is essential for price fixation based on color, texture, scent, hardness, durability, availability and rational use of available resources. This would also help in avoiding deception by timber traders.

There are two methods normally used for wood identification, viz., traditional approach and machine vision techniques. For many decades, traditional approaches have been instrumental in wood identification, like using $10 \times$ hand lenses to analyze the surface of the wood specimen in conjunction with their color, scent, hardness and weight. However for more reliable results, cross-sectional micro-structures of the wood samples are analysed in the laboratory and their features are compared with available samples of hardwood species for identification (Baas et al 1989).

The unavailability of xylarium microslides and literatures for comparing the microstructures of unidentified wood samples with known samples, and dearth of competent manpower in this field is the key challenge in wood identification. Further, imparting training to wood

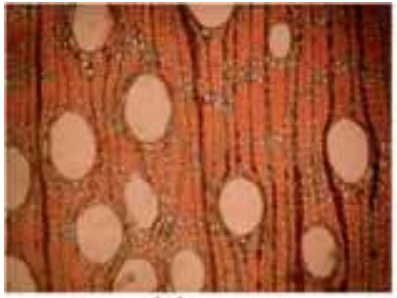

violaceus

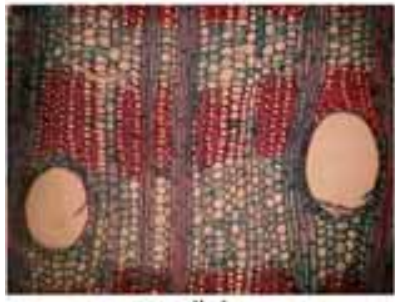

gomelleira

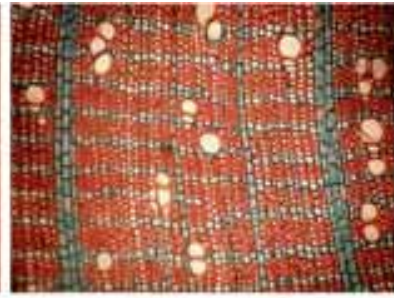

macrocarpa

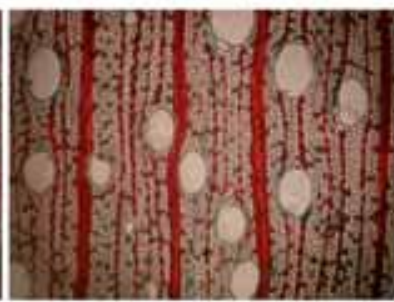

ovata

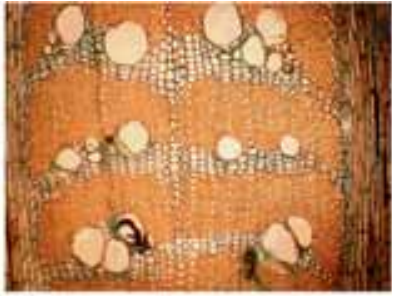

sp

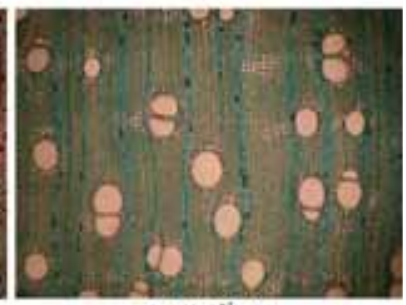

aurantium

Figure 1. Sample microscopic images of few hardwood species (Martins et al 2013). 
identification officers to get an expertise in identification of wood is a time-consuming process. Also, occupation as a wood certification officer is neither easy nor lucrative and possibility of unfairness and oversight cannot be denied. In the absence of systematic classification procedure for wood identification, a species has to be identified based on the combination of its microstructure features. There is massive hardwood diversity in tropical countries. India alone has over 1,200 hardwood species and, thus, remembering the microstructure of all the species is next to impossible. Recognition of large volumes of wood species, employing traditional approach is prolonged, erroneous and unfeasible sometimes. Therefore, to efficiently deal with the above mentioned issues researchers are looking into the possibility of coming up with computer assisted forest species/hardwood species identification system. Machine vision based systems outperform manual classifications when large volume of wood species is to be identified repeatedly with utmost accuracy, without getting fatigued. Thus, machine vision based wood identification techniques have emerged as an alternative in overcoming deficiencies associated with traditional approaches. The primary motivation behind employing machine vision based wood identification/classification system is the limitations associated with traditional approaches of wood identification.

\section{Literature review}

The machine vision techniques use classifiers to classify the wood images using their texture features. Various texture features of wood images have been extracted by means of GLCM (Tou et al 2007, 2009; Khalid et al 2008; Bremananth et al 2009; Wang et al 2010). Further, they have used different classifiers like MLP-BP-ANN, Pearson correlation, energy value, and SVM and achieved classification accuracy of $75.00 \%, 95.00 \%$, high accuracy, $80.00 \%$, and $91.70 \%$, respectively. The PCA, 2DPCA, (2D) ${ }^{2}$ PCA and LDA texture features have been used by You \& Cai (2009) for classification of wood species. The performance reported for LDA is a bit superior to PCA for cross-section images of wood, but inferior to 2DPCA and (2D) ${ }^{2}$ PCA.

Nasirzadeh et al (2010) used LBP variants for feature extraction and NN as a classifier. The LBP-HF has reported $96.60 \%$ accuracy compared to $91.00 \%$ recognition accuracy obtained by the traditional LBP ${ }^{\text {ri }}$. Yusof et al (2010) used combined features obtained by Gabor filter and GLCM techniques from macroscopic images and achieved a recognition rate of $90.33 \%$ for test dataset with MLP-ANN classifier. Further, Khairuddin et al (2011) employed BGLAM and SPPD to extract features. Before applying these features to the final classification stage, preclassification was carried out by K-means clustering followed by dimensionality reduction using LDA and KDA/GSVD. This scheme has accounted for a classification accuracy of $96.15 \%$ with K-NN classifier. The statistical features, namely, mean, SD, entropy and contrast have been obtained from wood stereogram images using a Gabor filter bank. The fusion of entropy, mean and SD features classified by NN classifier shows $94.58 \%$ recognition accuracy (Wang et al 2012).

Yusof et al (2013a, b) proposed a kernel genetic algorithm for selection of nonlinear features of macroscopic images of tropical wood species obtained by GLCM, BGLAM and SPPD techniques. This approach has brought in dimensionality reduction, and reported a classification accuracy of $98.69 \%$ with LDA classifier. Subsequently, a pre-classifier approach that uses fuzzy-logic concept was introduced by Yusof et al (2013a, b) with an aim to cluster the database. The experimental outcome reported around $93.00 \%$ accuracy compared to $88.90 \%$ accuracy obtained without incorporating fuzzy-logic pre-classifier. The ACA was employed by Ahmad \& Yusof (2013) to train and test the feature dataset obtained by BGLAM and SPPD techniques. A 
recognition rate of $96.75 \%$ was reported for experimental work performed using 24 clusters. Wang et al (2013) employed a MMI feature extraction technique to obtain features of wood stereogram images. Further, K-NN and SVM classifiers were investigated and a classification accuracy of $86.53 \%$ was achieved for statistical features of MMI with SVM classifier.

Martins et al (2013) presented a database of microscopic images of forest species that consist of 37 softwood and 75 hardwood species. Further, they obtained structural, GLCM and LBP features of these species, and a recognition accuracy of $98.60 \%$ and $86.00 \%$ was reported for two-class and multi-class (112) classification using SVM classifier. Afterwards, Cavalin et al (2013) extracted GLCM, LBP, and LPQ features from the images obtained by quad-tree decomposition method. The fusion of GLCM and LPQ features reported a recognition accuracy of 93.20\% using SVM classifier. The GLCM features obtained from Gabor wavelet images of hardwood species produced $92.60 \%$ classification accuracy with MLP-BP-NN classifier (Yadav et al 2013). Subsequently, Yadav et al (2014) acquired Coiflet DWT based features of the hardwood species. A recognition accuracy of $92.20 \%$ was reported for these features using MLP and logistic classifiers with 10-fold cross validation approach. Paula-Filho et al (2014) proposed a two level divide-and-conquer classification strategy to categorize the macroscopic images of 41 species using SVM classifier. Feature set obtained by a combination of several feature extraction techniques was classified with six number of classifiers and reported best recognition accuracy of $97.77 \%$.

A comprehensive analysis of the reviewed work shows that different texture features and classification algorithms are used for wood species identification (macroscopic, microscopic and stereogram images). The literature review discloses the fact that the texture feature information is extracted from single scale (original grayscale) images that could not acquire all the significant information of the images helpful in the efficient classification of hardwood species. Pan \& Kudo (2012) have stated that microscopic images of wood carry significant information useful in its precise identification as compared to limited information possessed by macroscopic images. Hence, current work utilizes microscopic images for hardwood species identification. Further, the classification accuracy of hardwood species can be improved either by incorporating appropriate texture feature extraction technique capable of obtaining discernible features of the image or by using suitable classifier. However, the classifiers performance highly depends on the quality of its input features. Thus, second motivation of the proposed methodology is extraction of the significant texture features of the microscopic images of hardwood species for their efficient classification. The DWT has been used here due to its multiresolution capability for analyzing images at different frequencies for several levels of resolutions (Mallat 1989). In the light of the above reasoning, the DWT based hybrid texture feature extraction technique capable of acquiring significant features of hardwood species has been proposed here. Three classifiers are incorporated to examine the efficacy of these techniques.

The contents of the research work are organized as follows: Section 3 gives detailed description of the proposed methodology for hardwood species classification using DWT based hybrid texture features and concise review of DWT, FOS, LBP variants, feature selection and classification algorithms. A critical discussion of experimental results is given in Section 4. Eventually, the work is concluded in Section 5.

\section{Proposed methodology}

The hardwood species classification using DWT based hybrid texture feature extraction techniques is illustrated in figure 2. The classification procedure is implemented in four major 


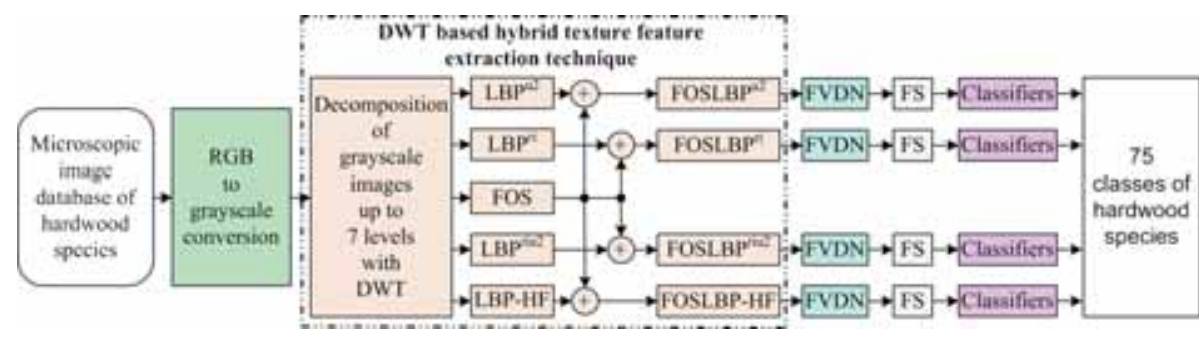

Figure 2. Block diagram of hardwood species classification with DWT based hybrid texture feature extraction techniques.

steps, namely, pre-processing, texture feature extraction, feature selection and classification. The individual blocks are concisely described as follows:

\subsection{Pre-processing}

As artificial color information is aided while preparing image samples of hardwood species to enhance its anatomical features, pre-processing step is employed to acquire grayscale images from color (RGB) images. The expression for RGB to gray scale conversion is given by (Kanan \& Cottrell 2012):

$$
G_{\text {luminance }}=0.2989^{*} R+0.5878^{*} G+0.1140^{*} B .
$$

where $G_{\text {luminance }}$ stands for grayscale image obtained by considering only luminance information; while eliminating the hue and saturation information of color image. The R, G and B stands for red, green and blue components of color image. This transformation facilitates significant reduction in computational time during feature extraction process.

\subsection{Texture feature extraction}

A 2D-DWT is a prevalent mathematical approach of hierarchical decomposition of an image, used in several applications such as face recognition, feature extraction, biomedical imaging and fingerprint anti-spoofing (Nikam \& Agarwal 2008). It provides the frequency and time information of a signal simultaneously, in an efficient manner (Livens et al 1997). The Daubechies wavelet family designed by Ingrid Daubechies has been chosen as a decomposition filter in 2D-DWT (Sripath 2003). The db3 wavelet of Daubechies family has been widely used in image analysis applications like face recognition (Wang et al 1998). The reason for selecting $\mathrm{db} 3$ as decomposition filter is its properties such as compact support, orthogonal, symmetrical behaviour and use of overlapping windows to show changes between all the pixel intensities (Jadhav \& Holambe 2009).

The DWT transformation is preferred for image decomposition due to its ability of detecting features at the unique resolution that may remain unnoticeable at any other resolution. The 2DDWT consists of a scaling function $\varphi(x, y)$ and three wavelet functions, $\psi^{H}(x, y), \psi^{V}(x, y)$ and $\psi^{D}(x, y)$, which are useful in measuring gray level variations in horizontal, vertical and diagonal directions, respectively. The 2D-DWT expression for a given image $f(x, y)$ of size $M \times N$ is given by Eqs. (2)-(5) (Gonzalez \& Woods 2011),

$$
\varphi_{j, r, c}(x, y)=2^{j / 2} \varphi\left(2^{j} x-r, 2^{j} y-c\right)
$$




$$
\psi_{j, r, c}^{i}(x, y)=2^{j / 2} \psi^{i} \varphi\left(2^{j} x-r, 2^{j} y-c\right), i=\{H, V, D\},
$$

where $\varphi$ and $\psi$ stands for scaled and translated basis functions, respectively.

$$
\begin{gathered}
W_{\varphi}\left(j_{0}, r, c\right)=\frac{1}{\sqrt{M N}} \sum_{x=0}^{M-1} \sum_{y=0}^{N-1} f(x, y) \varphi_{j_{0}, r, c}(x, y) \\
W_{\psi}^{i}(j, r, c)=\frac{1}{\sqrt{M N}} \sum_{x=0}^{M-1} \sum_{y=0}^{N-1} f(x, y) \psi_{j, r, c}^{i}(x, y), i=\{H, V, D\} .
\end{gathered}
$$

In Eq. (4) $j_{0}$ is an arbitrary starting scale. An approximation of $f(x, y)$ at $j_{0}$ scale is defined by $W_{\varphi}\left(j_{0}, r, c\right)$ coefficient, while diagonal, vertical and horizontal information for $j \geq j_{0}$ scale are given by $W_{\psi}^{i}(j, r, c)$ coefficients.

The FOS is one of the simplest and computationally efficient techniques for describing texture by using the intensity histogram of an image (Koenderink 1984). In FOS, the features that are taken into account are mean, SD, kurtosis and skewness. For a grayscale image, mean is a measure of the average intensity of pixels, while SD is a measure of contrast. Skewness is a measure of symmetry (it deals with the degree of histogram asymmetry around the mean) and kurtosis is the descriptor of the shape of probability distribution.

A first-order circular derivative pattern produced by combining the binary gradient directions is known as LBP, which considers an image is composed of micro-patterns. The distribution of edges and additional features in an image can be obtained from the micro-patterns (Nanni et al 2012; Doshi \& Schaefer 2012). The LBP is a dominant texture descriptor, extensively researched in content based image recognition, demographic classification and face recognition (Ojala et al 1994; Huang et al 2011). This method is reasonably simple, computationally efficient (Ojala et al 1996, 2002) and has an edge over several other feature descriptors. The LBP label for a centre pixel coordinate $(x, y)$ of an image is given by

$$
L B P_{P, R}(x, y)=\sum_{p=0}^{P-1} s\left(f(x, y)-f\left(x_{p}, y_{p}\right)\right) 2^{p},
$$

where $f(x, y)$ is gray value of the pixel of interest and $f\left(x_{p}, y_{p}\right)$ is gray value of $p^{t h}$ neighborhood of the centre pixel. The number of sampling points on circular neighborhood is denoted by $P$ and the spatial resolution of the neighborhood is represented by $R$. The $s(z)=\left\{\begin{array}{l}0, z<0 \\ 1, z \geq 0\end{array}\right\}$ represents the thresholding function.

A LBP pattern is called uniform pattern if no more than 2-bit wise transition (0 to 1 or 1 to 0 ) is reported in the LBP circular binary pattern (Ojala et al 2002). The $\mathrm{LBP}^{\mathrm{u} 2}$ histogram has separate bins for each uniform pattern and only single bin is assigned to the entire nonuniform patterns. The uniform patterns account for nearly $90.00 \%$ of the whole pattern with $(8,1)$ neighborhood and close to $70 \%$ for $(16,2)$ neighborhood for texture images (Pietikäinen et al 2011). The $\mathrm{LBP}^{\mathrm{u} 2}$ has produced superior recognition result for various applications and is statistically robust. Rotation of an image produces diverse LBP codes, to address this issue LBP ${ }^{\text {ri }}$ has been proposed by Ojala et al (2002). The LBPri is generated by circularly rotating the LBP code until its minimum value is achieved. This descriptor produces overall 36 bin histograms 
for each image due to 36 unlike 8-bit rotation invariant codes (Pietikäinen et al 2000; Mäenpää 2003).

The performance of $\mathrm{LBP}^{\mathrm{ri}}$ is poor because (1) the occurrence frequency of 36 individual patterns included in $\mathrm{LBP}^{\mathrm{ri}}$ varies to a great extent, and (2) the crude quantization of the angular spaces at an interval of $45^{\circ}$. To overcome aforesaid issues, the LBPriu2 has been proposed (Mäenpää 2003). The LBP riu2 operator has $P+1$ uniform patterns in a circular neighborhood of $P$ pixels, while a single label is assigned to all the non-uniform patterns. Ahonen et al (2009) have proposed LBP-HF, which is invariant to the rotary motion of an image and it retains the most discriminative characteristics. The LBP-HF is realized by taking the discrete Fourier transform of $\mathrm{LBP}^{\mathrm{u} 2}$ (Zhao et al 2012). It is different from several rotation invariant histogram based texture feature techniques, because LBP-HF features are calculated from the histogram of the entire image region (globally), rather than calculating for each pixel (locally).

The texture feature extraction is the most significant stage in the hardwood species classification process. Initially, the grayscale images are decomposed up to the seven (1-7) levels by DWT using Daubechies (db3) wavelet as decomposition filter. At the $1^{\text {st }}$ level of image decomposition, the grayscale image is subdivided into four identical, quarter-size subimages, namely, approximation (LL1), horizontal (LH1), vertical (HL1) and diagonal (HH1) components. Thereafter, the LL1 component is decomposed into four equal quarter-size subimages (LL2, LH2, HL2 and HH2) at the $2^{\text {nd }}$ level of image decomposition. The process of partitioning the approximation component is repeated till the required level of image decomposition has been reached. As an illustration, the color image of pachycarpa species is illustrated in figure 3(a), whereas DWT based $5^{\text {th }}$ level image decomposition of the grayscale image of pachycarpa species is shown in figure 3(b). It is evident that each scale subimages have distinctive information.

Subsequently, five texture descriptors are employed to extract distinct features from each of the subimages at different (1-7) levels of image decomposition. The texture feature descriptors used in this work are, FOS and four variants of $\mathrm{LBP}\left(\mathrm{LBP}^{\mathrm{u} 2}, \mathrm{LBP}^{\mathrm{ri}}, \mathrm{LBP}^{\text {riu2 }}\right.$ and LBP-HF). The FOS features are chosen as they reveal the global features. The LBP variants are selected to depict the local texture descriptors of the image. Further, at each level of image decomposition the FOS features are concatenated with LBP variants features to construct feature vector data.

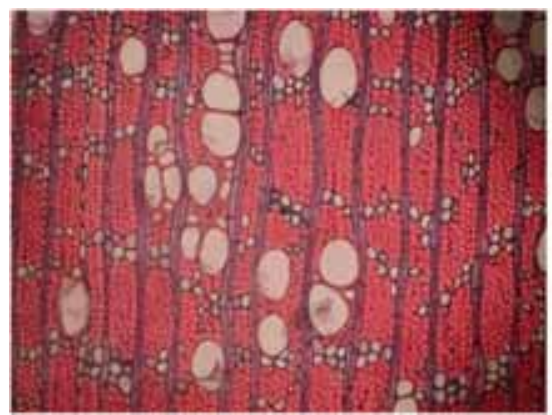

(a)

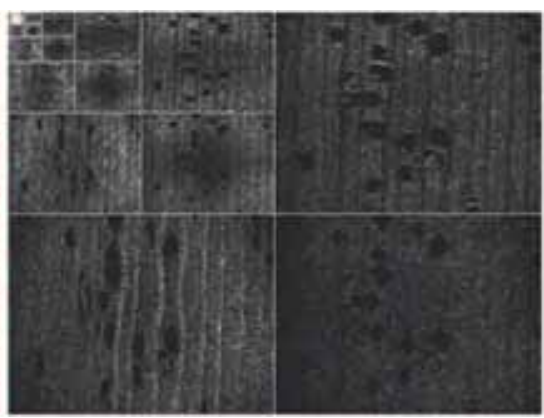

(b)

Figure 3. (a) Color image of pachycarpa species, (b) the pachycarpa species grayscale image obtained at the $5^{\text {th }}$ level of image decomposition using DWT. 
The expression for obtaining DWTFOSLBP feature vector data for each image at the $L^{\text {th }}$ level of image decomposition is given by the following equation:

$$
\begin{aligned}
& \text { DWTFOSLBP } \\
& =\sum_{l=1}^{L}\left[\begin{array}{l}
\left(L B P_{P, R}\left(W_{\varphi}^{l}\right)+L B P_{P, R}\left(W_{\psi}^{H^{l}}\right)+L B P_{P, R}\left(W_{\psi}^{V^{l}}\right)+L B P_{P, R}\left(W_{\psi}^{D^{l}}\right)\right) \\
+\left(m\left(W_{\varphi}^{l}\right)+m\left(W_{\psi}^{H^{l}}\right)+m\left(W_{\psi}^{V^{l}}\right)+m\left(W_{\psi}^{D^{l}}\right)\right) \\
+\left(\sigma\left(W_{\varphi}^{l}\right)+\sigma\left(W_{\psi}^{H^{l}}\right)+\sigma\left(W_{\psi}^{V^{l}}\right)+\sigma\left(W_{\psi}^{D^{l}}\right)\right) \\
+\left(\mu_{3}\left(W_{\varphi}^{l}\right)+\mu_{3}\left(W_{\psi}^{H^{l}}\right)+\mu_{3}\left(W_{\psi}^{V^{l}}\right)+\mu_{3}\left(W_{\psi}^{D^{l}}\right)\right) \\
+\left(\mu_{4}\left(W_{\varphi}^{l}\right)+\mu_{4}\left(W_{\psi}^{H^{l}}\right)+\mu_{4}\left(W_{\psi}^{V^{l}}\right)+\mu_{4}\left(W_{\psi}^{D^{l}}\right)\right)
\end{array}\right]
\end{aligned}
$$

where $L$ stands for number of image decomposition levels and $L B P_{P, R}$ represent one of the LBP variants (i.e., $\mathrm{LBP}^{\mathrm{u} 2}$, $\mathrm{LBP}^{\mathrm{ri}}$, $\mathrm{LBP}^{\text {riu2 }}$ and LBP-HF) features. The $m, \sigma, \mu_{3}$ and $\mu_{4}$ represent mean, SD, kurtosis and skewness features of FOS, respectively. Thus, on the basis of integration of DWT with FOS and LBP variants, four hybrid texture feature extraction techniques namely, DWTFOSLBP ${ }^{\mathrm{u} 2}$, DWTFOSLBP ${ }^{\mathrm{ri}}$, DWTFOSLBP ${ }^{\text {riu2 }}$ and DWTFOSLBP-HF have been proposed. The feature vector dataset produced by these techniques have different range of values, which is normalized in the range $0-1$, thus rendering it in the form useful as an input to the classifier. The expression for FVDN is given by

$$
F_{\text {Norm }}=\left(\frac{F-\min (F)}{\max (F)-\min (F)}\right) .
$$

where $F_{N o r m}$ : normalized feature vector data, and $F$ : original feature vector data.

\subsection{Feature selection (FS)}

The key objective of FS technique is to eliminate the irrelevant features, and retain a subset of features that efficiently describe the observed input data. The FS facilitates in reducing the effect of curse of dimensionality, gives better insight of data, and improves the performance of classifier by using a subset of features as input (Chandrashekar \& Sahin 2014). The FS techniques are categorized into filter, wrapper and embedded methods in the context of classification (Saeys et al 2007). The filter techniques are fast, computationally efficient and independent of classification algorithms. The mRMR feature selection technique based on mutual information quotient proposed by Peng et al (2005) is a multivariate filter technique has been chosen in this work.

Since, the proposed feature extraction techniques generate a large number of complex features, of which many features can be redundant or less significant. Therefore, mRMR feature selection technique is used in the third stage to select best feature subsets helpful in the discrimination of hardwood species.

\subsection{Classifiers}

In the fourth stage, the effectiveness of the DWT based hybrid texture feature extraction techniques for the classification of hardwood species has been assessed by three classifiers (linear SVM, RBF kernel SVM and RF classifier). The SVM is widely used machine learning algorithm employed in several pattern recognition problems in image processing (Yaghouby \& Ayatollahi 2010; Chowdhury et al 2011). It was initially proposed to handle binary 
classification task (Cortes \& Vapnik 1995). The binary SVM classifier is later on extended for multi-class SVM classification using methods such as "one against one", "one against all", and "directed acyclic graph" (Platt et al 1999).

Linear SVM classifier directly works on the given data in the original input space and is found suitable for a dataset with large number of features (Yuan et al 2012; Fan et al 2008). Linear SVM classifier is considered to be efficient and enjoys faster training and testing procedure (Yuan et al 2012). It has used multi-class SVM approach proposed by Crammer \& Singer (2002). The RBF is one of the most popular nonlinear kernel function (Shan et al 2009; Chang \& Lin 2011) used in classification. The RBF kernel SVM maps the original input data into a high-dimensional space, wherein mapped data are linearly separable. The RF classifier is an ensemble machine learning technique, which builds several classification and regression trees by incorporating additional layer of randomness to bagging (Breiman 2001). It has been widely used as a classifier in bioinformatics, geology and pattern recognition (Coussement \& Van den Poel 2008). It is robust against over fitting, handles large dataset, computationally efficient, and easy to implement.

The effectiveness of the DWT based hybrid texture feature extraction techniques has been observed on the basis of the classification accuracy obtained through the classifiers. Consequently, the best combination of DWT based hybrid texture features and the classifier is identified on the basis of the best classification accuracy.

\section{Results and discussion}

The foremost objective of this research work has been to improve the distinct texture feature extraction capability of FOS and LBP variants with proposed DWT based hybrid texture feature extraction techniques. The emphasis in this work has been given to assess the efficiency of DWT based hybrid texture feature extraction techniques for the classification of microscopic image database of 75 hardwood species using different classifiers (Alexandre 2010). Therefore, the classifiers (linear SVM, RBF kernel SVM and RF) have been selected based on their general performance characteristics. These classifiers are being examined by means of MATLAB implementations of LibLINEAR (Fan et al 2008), LIBSVM (Chang \& Lin 2011) and RF_MexStandalone-v0.02 (Jaiantilal 2010), respectively. The Windows 7 professional operating system machine equipped with Intel(R) Core(TM), i7-2600 CPU @ 3.40 GHz processor and 16 GB RAM has been employed to accomplish the task.

\subsection{Hardwood species database}

The present work uses an open access database of microscopic images of 75 hardwood species, and 20 samples of each species making it in total as 1,500 samples. The sample collection and preparation process is available in (Martins et al 2013). Each of these species is correctly labelled by the experts in the laboratory of wood anatomy at Federal University of Parana, Curitiba, Brazil. The hardwood species used in this work are listed in table 1.

\subsection{Parameter selection}

The image pre-processing and texture feature extraction task have been performed using MATLAB R2013a environment. The state-of-the-art texture features are obtained using available MATLAB codes of LPQ (Ojansivu \& Heikkilä 2008) and LBP variants (http://www.cse.oulu.fi/ 
Table 1. List of hardwood species.

\begin{tabular}{|c|c|c|c|c|c|c|c|}
\hline $\begin{array}{l}\text { Sr. } \\
\text { no. }\end{array}$ & Family & Gender & Species & $\begin{array}{l}\text { Sr. } \\
\text { no. }\end{array}$ & Family & Gender & Species \\
\hline 1 & Ephedraceae & Ephedra & californica & 39 & Lauraceae & Nectandra & $\mathrm{sp}$ \\
\hline 2 & Lecythidaceae & Cariniana & estrellensis & 40 & Lauraceae & Ocotea & porosa \\
\hline 3 & Lecythidaceae & Couratari & $\mathrm{sp}$ & 41 & Lauraceae & Persea & racemosa \\
\hline 4 & Lecythidaceae & Eschweilera & matamata & 42 & Annonaceae & Porcelia & macrocarpa \\
\hline 5 & Lecythidaceae & Eschweleira & chartaceae & 43 & Magnoliaceae & Magnolia & grandiflora \\
\hline 6 & Sapotaceae & Chrysophyllum & $\mathrm{sp}$ & 44 & Magnoliaceae & Talauma & ovata \\
\hline 7 & Sapotaceae & Micropholis & guianensis & 45 & Melastomataceae & Tibouchiana & sellowiana \\
\hline 8 & Sapotaceae & Pouteria & pachycarpa & 46 & Myristicaceae & Virola & oleifera \\
\hline 9 & Fabaceae-Cae. & Copaifera & trapezifolia & 47 & Myrtaceae & Campomanesia & xanthocarpa \\
\hline 10 & Fabaceae-Cae. & Eperua & falcata & 48 & Myrtaceae & Eucalyptus & globulus \\
\hline 11 & Fabaceae-Cae. & Hymenaea & courbaril & 49 & Myrtaceae & Eucalyptus & grandis \\
\hline 12 & Fabaceae-Cae. & Hymenaea & $\mathrm{sp}$ & 50 & Myrtaceae & Eucalyptus & saligna \\
\hline 13 & Fabaceae-Cae. & Schizolobium & parahyba & 51 & Myrtaceae & Myrcia & racemulosa \\
\hline 14 & Fabaceae-Fab. & Pterocarpus & violaceus & 52 & Vochysiaceae & Erisma & uncinatum \\
\hline 15 & Fabaceae-Mim. & Acacia & tucunamensis & 53 & Vochysiaceae & Qualea & $\mathrm{sp}$ \\
\hline 16 & Fabaceae-Mim. & Anadenanthera & colubrina & 54 & Vochysiaceae & Vochysia & laurifolia \\
\hline 17 & Fabaceae-Mim. & Anadenanthera & peregrina & 55 & Proteaceae & Grevillea & robusta \\
\hline 18 & Fabaceae-Fab. & Dalbergia & jacaranda & 56 & Proteac & Grevillea & $\mathrm{sp}$ \\
\hline 19 & Fabaceae-Fab. & Dalbergia & spruceana & 57 & Proteaceae & Roupala & $\mathrm{sp}$ \\
\hline 20 & Fabaceae-Fab. & Dalbergia & variabilis & 58 & Moraceae & Bagassa & guianensis \\
\hline 21 & Fabaceae-Mim. & Dinizia & excelsa & 59 & Moraceae & Brosimum & alicastrum \\
\hline 22 & Fabaceae-Mim. & Enterolobium & schomburgkii & 60 & Moraceae & Ficus & gomelleira \\
\hline 23 & Fabaceae-Mim. & Inga & sessilis & 61 & Rhamnaceae & Hovenia & dulcis \\
\hline 24 & Fabaceae-Mim. & Leucaena & leucocephala & 62 & Rhamnaceae & Rhamnus & frangula \\
\hline 25 & Fabaceae-Fab. & Lonchocarpus & subglaucescens & 63 & Rosaceae & Prunus & sellowii \\
\hline 26 & Fabaceae-Mim. & Mimosa & bimucronata & 64 & Rosaceae & Prunus & serotina \\
\hline 27 & Fabaceae-Mim. & Mimosa & scabrella & 65 & Rubiaceae & Faramea & occidentalis \\
\hline 28 & Fabaceae-Fab. & Ormosia & excelsa & 66 & Meliaceae & Cabralea & canjerana \\
\hline 29 & Fabaceae-Mim. & Parapiptadenia & rigida & 67 & Meliaceae & Carapa & guianensis \\
\hline 30 & Fabaceae-Mim. & Parkia & multijuga & 68 & Meliaceae & Cedrela & fissilis \\
\hline 31 & Fabaceae-Mim. & Piptadenia & excelsa & 69 & Meliaceae & Khaya & ivorensis \\
\hline 32 & Fabaceae-Mim. & Pithecellobium & jupunba & 70 & Meliaceae & Melia & azedarach \\
\hline 33 & Rubiaceae & Psychotria & carthagenensis & 71 & Meliaceae & Swietenia & macrophylla \\
\hline 34 & Rubiaceae & Psychotria & longipes & 72 & Rutaceae & Balfourodendron & riedelianum \\
\hline 35 & Bignoniaceae & Tabebuia rosea & alba & 73 & Rutaceae & Citrus & aurantium \\
\hline 36 & Bignoniaceae & Tabebuia & $\mathrm{sp}$ & 74 & Rutaceae & Fagara & rhoifolia \\
\hline 37 & Oleaceae & Ligustrum & lucidum & 75 & Simaroubaceae & Simarouba & amara \\
\hline 38 & Lauraceae & Nectandra & rigida & & & & \\
\hline
\end{tabular}

CMV/Downloads/LBP), GLCM (Uppuluri 2010) and Gabor filter (Haghighat et al 2013). The $P$ and $R$ parameter values are considered as 8 and 1 , respectively, for all the variants of LBP because these values have yielded into fast and accurate feature extraction (Ojala et al 2002; Heikkilä et al 2009; Alexandre 2010). Total 18 features are calculated for each of the image with GLCM, using neighborhood distance of nine in four directions viz., $0^{\circ}, 45^{\circ}, 90^{\circ}$ and $135^{\circ}$. The 18 features of GLCM consist of f1-f13 features proposed by Haralick et al (1973) and five features proposed by Soh \& Tsatsoulis (1999) viz., autocorrelation, dissimilarity, cluster 
shade, cluster prominence and maximum probability. To obtain Gabor texture features, each of the images is processed by Gabor filter (five scales and eight directions) to generate 40 Gabor images. Further, from these images mean, SD and entropy features are calculated. To extract color image texture features, the R, G, and B components of each color image are concatenated to form a $M \times 3 N$ grayscale image, where size of each component is $M \times N$. Then, the features are extracted from a $M \times 3 N$ grayscale image to produce RGB image texture features. The authors have developed codes for DWT based hybrid texture feature extraction techniques in MATLAB using the $P$ and $R$ parameter values of LBP as 8 and 1 .

The optimum value of cost $(C)$ parameter for linear SVM classifier has been selected with linear search method in the range $\left(10^{-4}, 10^{-3}, \ldots, 10^{+5}\right)$, while the optimum value of $C$ and gamma $(\gamma)$ has been selected by using grid search method in the range $\left(10^{-4}, 10^{-3}, \ldots, 10^{+5}\right)$ for RBF kernel SVM classifier (Chu et al 2013). The tolerance of termination criteria ( $\xi$ ) has been tested for several values $(0.1,0.01,0.001,0.0001)$ and it was found that $\xi=0.01$ gives best trade-off between classification accuracy and computational time. The RF classifier needs to optimize number of trees ( $n$ Tree) and number of randomly chosen features ( $m \operatorname{Tr} y$ ) for generating a prediction model. Typically 500-2,000 trees are grown and the results aggregated by averaging (Prasad et al 2006). The authors have varied the $n$ Tree values between 100 and 1000 and found that addition of number of trees beyond 450-500 was not having significant improvement in the classification accuracy for our dataset. Thus as a trade-off between classification accuracy and computational time $n$ Tree $=500$ (Ai et al 2014; Liu et al 2013) and $m \operatorname{Tr} y=$ floor $(\sqrt{\text { number of features }})$ (DiFranco et al 2011; Ai et al 2014) has been selected in this experimentation. These set of parameters are used throughout Section 4 for the analytical work.

\subsection{Evaluation procedure}

Two different strategies have been adopted to test the effectiveness of the state-of-the-art and proposed DWT based hybrid texture features extracted from grayscale and RGB images of hardwood species, categorized under: (1) the 10-fold cross validation approach and (2) randomly divided database.

4.3a The 10-fold cross validation: Here, the classification task employs 10-fold cross validation (Geisser 1993) to obtain the end result by dividing the texture feature dataset into 10 equal-size folds. The major reason for using 10-fold cross validation is to ensure that results remain unbiased for a given partitioned dataset. Out of 10-folds, 9-folds are used for training and remaining 1-fold is used for testing. Thus, $90 \%$ data is used for training and $10 \%$ data is used for testing. Further, the process is repeated 10 times so that each sample is used as test data. The final classification accuracy is the average of all the 10 results.

4.3b Randomly divided database: In this case, the total available dataset is randomly divided into fixed training and testing subsets for each individual species. The investigations have been carried out with four different proportions of training and testing datasets which are listed below:

1. $80 \%$ data for training and $20 \%$ data for testing $(80 / 20)$

2. $70 \%$ data for training and $30 \%$ data for testing $(70 / 30)$

3. $60 \%$ data for training and $40 \%$ data for testing (60/40)

4. $50 \%$ data for training and $50 \%$ data for testing $(50 / 50)$ 


\subsection{Performance evaluation of texture feature extraction techniques using 10-fold cross validation}

The classification accuracy obtained for full feature vector data and reduced feature vector data by mRMR method using 10-fold cross validation is as follows:

4.4a State-of-the-art texture features using full feature vector data: The classification accuracy for state-of-the-art texture features obtained from grayscale and RGB images is presented in table 2. Of all the methods, Gabor texture features achieved highest classification accuracy of $93.47 \pm 1.43 \%$ and $92.33 \pm 1.70 \%$ for grayscale and RGB images, respectively, using linear SVM classifier. It was also observed that RBF kernel SVM produced results $(90.20 \pm 3.25 \%$ and $90.53 \pm 2.47 \%$ ) comparable to linear SVM classifier, whereas the performance of RF classifiers was comparatively poor $(77.80 \pm 3.14 \%$ and $81.27 \pm 1.85 \%)$.

4.4b State-of-the-art texture features using feature vector data selected by mRMR technique: Using a subset of feature vector data for grayscale and RGB images obtained by the state-ofthe-art texture features is listed in table 3. The Gabor texture features classified by linear SVM classifier have again given best classification accuracy of $93.67 \pm 1.64 \%$ (100 features) and 92.33 $\pm 1.76 \%$ (115 features) for grayscale and RGB images, respectively. The results obtained by RBF kernel SVM $(91.07 \pm 2.39 \%$ and $91.33 \pm 2.63 \%)$ are comparable to linear SVM classifier, while RF classifier performed poorly $(79.93 \pm 2.36 \%$ and $82.93 \pm 2.61 \%)$.

4.4c Proposed DWT based hybrid texture features using full feature vector data: The classification accuracy achieved by DWT based hybrid texture feature extraction techniques for grayscale image up to the $7^{\text {th }}$ level of image decomposition is listed in table 4 . Amongst

Table 2. Classification accuracy achieved using full feature vector data of state-of-the-art texture features obtained from grayscale and RGB images.

\begin{tabular}{|c|c|c|c|c|c|}
\hline \multirow{2}{*}{$\begin{array}{l}\text { Image } \\
\text { type }\end{array}$} & \multirow[b]{2}{*}{ Technique } & \multicolumn{4}{|c|}{$\% \mathrm{CA} \pm \mathrm{SD}$ achieved by classifiers } \\
\hline & & $\overline{\mathrm{NoF}}$ & Linear SVM & RBF kernel SVM & RF \\
\hline \multirow[t]{8}{*}{ Grayscale } & $\mathrm{LBP}^{\mathrm{u} 2}$ & 59 & $79.73 \pm 2.88$ & $81.47 \pm 2.64$ & $69.27 \pm 2.52$ \\
\hline & LBP $^{r i}$ & 36 & $77.33 \pm 2.86$ & $79.07 \pm 3.31$ & $53.47 \pm 2.98$ \\
\hline & LBPriu2 & 10 & $63.53 \pm 3.16$ & $67.07 \pm 2.71$ & $41.60 \pm 4.23$ \\
\hline & LBP-HF & 38 & $71.60 \pm 1.51$ & $71.40 \pm 1.49$ & $65.47 \pm 3.20$ \\
\hline & FOS & 4 & $15.80 \pm 2.59$ & $38.67 \pm 2.08$ & $33.13 \pm 2.72$ \\
\hline & GLCM & 72 & $80.47 \pm 3.32$ & $73.67 \pm 3.25$ & $53.93 \pm 2.74$ \\
\hline & LPQ & 256 & $90.80 \pm 2.13$ & $87.00 \pm 2.18$ & $70.53 \pm 1.96$ \\
\hline & Gabor & 120 & $93.47 \pm 1.43$ & $90.20 \pm 3.25$ & $77.80 \pm 3.14$ \\
\hline \multirow[t]{8}{*}{ RGB } & $\mathrm{LBP}^{\mathrm{u} 2}$ & 59 & $82.93 \pm 2.80$ & $83.93 \pm 2.25$ & $72.20 \pm 2.76$ \\
\hline & $\mathrm{LBP}^{\mathrm{ri}}$ & 36 & $81.93 \pm 1.19$ & $82.93 \pm 1.94$ & $59.00 \pm 4.71$ \\
\hline & LBP ${ }^{\text {riu2 }}$ & 10 & $66.00 \pm 2.80$ & $71.53 \pm 2.44$ & $45.80 \pm 3.43$ \\
\hline & LBP-HF & 38 & $75.67 \pm 2.34$ & $76.07 \pm 3.05$ & $70.00 \pm 3.64$ \\
\hline & FOS & 4 & $23.73 \pm 2.09$ & $49.47 \pm 2.49$ & $44.73 \pm 3.30$ \\
\hline & GLCM & 72 & $82.33 \pm 2.07$ & $80.00 \pm 1.77$ & $63.60 \pm 2.56$ \\
\hline & LPQ & 256 & $91.80 \pm 1.30$ & $87.33 \pm 1.54$ & $72.80 \pm 2.43$ \\
\hline & Gabor & 120 & $92.33 \pm 1.70$ & $90.53 \pm 2.47$ & $81.27 \pm 1.85$ \\
\hline
\end{tabular}


Table 3. Classification accuracy achieved using mRMR based reduced feature vector data of state-of-theart texture features obtained from grayscale and RGB images.

\begin{tabular}{|c|c|c|c|c|c|c|c|}
\hline \multirow[b]{2}{*}{ Image type } & \multirow[b]{2}{*}{ Technique } & \multicolumn{6}{|c|}{$\% \mathrm{CA} \pm \mathrm{SD}$ achieved by classifiers } \\
\hline & & $\overline{\mathrm{NoF}}$ & Linear SVM & $\mathrm{NoF}$ & RBF kernel SVM & $\mathrm{NoF}$ & $\mathrm{RF}$ \\
\hline \multirow[t]{8}{*}{ Grayscale } & $\mathrm{LBP}^{\mathrm{u} 2}$ & 55 & $76.93 \pm 3.31$ & 55 & $79.73 \pm 2.54$ & 55 & $69.07 \pm 3.59$ \\
\hline & LBPri & 35 & $76.33 \pm 2.51$ & 35 & $78.53 \pm 3.01$ & 30 & $54.26 \pm 4.36$ \\
\hline & LBP ${ }^{\text {riu2 }}$ & 9 & $59.80 \pm 3.76$ & 9 & $64.67 \pm 2.96$ & 9 & $42.67 \pm 4.23$ \\
\hline & LBP-HF & 37 & $71.80 \pm 2.37$ & 37 & $71.13 \pm 1.89$ & 37 & $64.40 \pm 5.28$ \\
\hline & FOS & 4 & $16.73 \pm 1.79$ & 4 & $36.20 \pm 2.93$ & 4 & $34.47 \pm 3.81$ \\
\hline & GLCM & 60 & $80.80 \pm 3.45$ & 60 & $73.47 \pm 3.05$ & 70 & $55.27 \pm 4.30$ \\
\hline & LPQ & 255 & $90.67 \pm 1.94$ & 175 & $86.87 \pm 2.11$ & 175 & $72.53 \pm 2.72$ \\
\hline & Gabor & 100 & $93.67 \pm 1.64$ & 100 & $91.07 \pm 2.39$ & 75 & $79.93 \pm 2.36$ \\
\hline \multirow[t]{8}{*}{ RGB } & $\mathrm{LBP}^{\mathrm{u} 2}$ & 55 & $82.47 \pm 2.11$ & 50 & $83.33 \pm 2.45$ & 50 & $72.47 \pm 3.39$ \\
\hline & $\mathrm{LBP}^{\mathrm{ri}}$ & 35 & $82.00 \pm 1.26$ & 35 & $82.87 \pm 1.94$ & 30 & $58.93 \pm 3.17$ \\
\hline & LBPriu2 & 9 & $59.00 \pm 2.50$ & 9 & $65.67 \pm 2.70$ & 9 & $43.80 \pm 3.28$ \\
\hline & LBP-HF & 37 & $75.73 \pm 2.14$ & 37 & $75.80 \pm 3.36$ & 37 & $69.73 \pm 4.81$ \\
\hline & FOS & 4 & $23.93 \pm 2.14$ & 4 & $47.53 \pm 3.36$ & 4 & $44.47 \pm 3.11$ \\
\hline & GLCM & 70 & $82.73 \pm 2.11$ & 60 & $80.07 \pm 1.87$ & 70 & $63.00 \pm 3.76$ \\
\hline & LPQ & 250 & $91.53 \pm 1.22$ & 225 & $87.20 \pm 1.62$ & 175 & $73.40 \pm 3.70$ \\
\hline & Gabor & 115 & $92.33 \pm 1.76$ & 100 & $91.33 \pm 2.63$ & 100 & $82.93 \pm 2.61$ \\
\hline
\end{tabular}

the proposed techniques DWTFOSLBP ${ }^{\mathrm{u} 2}$ and DWTFOSLBP ${ }^{\text {riu2 }}$ texture features have achieved highest classification accuracy of $97.67 \pm 0.79 \%$ using linear SVM classifier. These accuracies are observed at $4^{\text {th }}$ and $5^{\text {th }}$ level of image decomposition with 1008 and 280 features for DWTFOSLBP ${ }^{\mathrm{u} 2}$ and DWTFOSLBP ${ }^{\text {riu2 }}$ techniques, respectively. The DWTFOSLBP ${ }^{\mathrm{ri}}$ has obtained a classification accuracy of $96.73 \pm 1.24 \%$ (640 features) at the $4^{\text {th }}$ level of image decomposition with linear SVM classifier. The DWTFOSLBP-HF has resulted into maximum classification accuracy of $96.60 \pm 1.46 \%$ (1008 features) at the $6^{\text {th }}$ level of image decomposition by linear SVM classifier.

Further, the highest classification accuracy of $97.67 \pm 1.14 \%$ has been achieved by DWTFOSLBP ${ }^{\mathrm{u} 2}$ texture features at the $5^{\text {th }}$ level of image decomposition, when classified with RBF kernel SVM classifier. This accuracy is at par with the accuracy achieved by linear SVM classifier with minor variation of $0.35 \%$ in the SD value. The maximum classification accuracy produced by RF classifier is $95.27 \pm 1.87 \%$ for DWTFOSLBP-HF features at the $6^{\text {th }}$ level of image decomposition. Thus, for grayscale images, DWT based hybrid texture features have produced maximum classification accuracy for both linear SVM and RBF kernel SVM classifier, whereas RF classifier gave comparatively poor classification accuracy.

The classification accuracy achieved by the classifiers using RGB image features acquired by DWT based hybrid texture feature extraction techniques is better than those of grayscale images, as is evident from table 5. Amongst the proposed techniques, the highest classification accuracy of $98.40 \pm 0.64 \%$ has been recorded for DWTFOSLBP ${ }^{\mathrm{u}}$ texture features at the $4^{\text {th }}$ level of image decomposition by linear SVM classifier. This technique has also given a comparable classification accuracy of $98.13 \pm 0.88 \%$ at the $4^{\text {th }}$ level of image decomposition with RBF kernel SVM classifier. This improvement is also visible in the classification accuracy achieved for the texture features with RF classifier. The other proposed techniques have also shown improvement in the classification accuracy for texture features obtained from RGB images as is evident from table 5 . 
Table 4. Classification accuracy achieved using full feature vector data of DWT based hybrid texture features extracted from grayscale images.

\begin{tabular}{|c|c|c|c|c|c|}
\hline \multirow[b]{2}{*}{ Technique } & \multirow[b]{2}{*}{ IDL } & \multicolumn{4}{|c|}{$\% \mathrm{CA} \pm \mathrm{SD}$ achieved by classifiers } \\
\hline & & $\overline{\mathrm{NoF}}$ & Linear SVM & RBF kernel SVM & RF \\
\hline \multirow[t]{7}{*}{ DWTFOSLBP ${ }^{\mathrm{u} 2}$} & 1 & 252 & $90.67 \pm 1.81$ & $90.33 \pm 2.07$ & $82.60 \pm 3.05$ \\
\hline & 2 & 504 & $95.73 \pm 1.38$ & $94.33 \pm 1.55$ & $88.93 \pm 2.54$ \\
\hline & 3 & 756 & $97.13 \pm 1.51$ & $96.33 \pm 1.64$ & $93.20 \pm 1.25$ \\
\hline & 4 & 1008 & $97.67 \pm 0.79$ & $97.33 \pm 1.18$ & $94.13 \pm 1.17$ \\
\hline & 5 & 1260 & $97.33 \pm 1.09$ & $97.67 \pm 1.14$ & $94.47 \pm 1.09$ \\
\hline & 6 & 1512 & $97.07 \pm 1.00$ & $96.93 \pm 1.30$ & $95.00 \pm 1.23$ \\
\hline & 7 & 1764 & $96.73 \pm 1.24$ & $96.67 \pm 1.44$ & $94.13 \pm 1.08$ \\
\hline \multirow[t]{7}{*}{ DWTFOSLBP $^{\text {ri }}$} & 1 & 160 & $84.87 \pm 2.65$ & $87.07 \pm 2.40$ & $81.53 \pm 2.20$ \\
\hline & 2 & 320 & $92.73 \pm 2.05$ & $92.87 \pm 2.53$ & $88.13 \pm 1.85$ \\
\hline & 3 & 480 & $95.73 \pm 1.23$ & $95.73 \pm 1.78$ & $92.27 \pm 1.18$ \\
\hline & 4 & 640 & $96.73 \pm 1.24$ & $96.53 \pm 1.36$ & $93.73 \pm 1.38$ \\
\hline & 5 & 800 & $96.47 \pm 1.00$ & $96.33 \pm 1.48$ & $94.13 \pm 1.47$ \\
\hline & 6 & 960 & $96.07 \pm 1.49$ & $96.13 \pm 1.96$ & $94.00 \pm 1.41$ \\
\hline & 7 & 1120 & $95.40 \pm 1.46$ & $95.53 \pm 2.03$ & $94.33 \pm 1.34$ \\
\hline \multirow[t]{7}{*}{ DWTFOSLBP ${ }^{\text {riu2 }}$} & 1 & 56 & $86.00 \pm 3.10$ & $87.20 \pm 1.88$ & $79.73 \pm 3.59$ \\
\hline & 2 & 112 & $92.87 \pm 1.94$ & $92.60 \pm 1.52$ & $86.07 \pm 1.97$ \\
\hline & 3 & 168 & $96.60 \pm 0.66$ & $96.07 \pm 1.02$ & $91.00 \pm 1.81$ \\
\hline & 4 & 224 & $97.53 \pm 0.71$ & $97.13 \pm 1.69$ & $93.73 \pm 1.05$ \\
\hline & 5 & 280 & $97.67 \pm 0.79$ & $97.53 \pm 0.83$ & $94.67 \pm 1.47$ \\
\hline & 6 & 336 & $97.40 \pm 1.11$ & $97.13 \pm 1.37$ & $94.80 \pm 1.43$ \\
\hline & 7 & 392 & $96.07 \pm 1.59$ & $96.73 \pm 1.46$ & $94.67 \pm 1.47$ \\
\hline \multirow[t]{7}{*}{ DWTFOSLBP-HF } & 1 & 168 & $84.53 \pm 2.41$ & $85.47 \pm 3.94$ & $82.87 \pm 3.14$ \\
\hline & 2 & 336 & $91.80 \pm 1.48$ & $92.33 \pm 1.64$ & $89.20 \pm 1.60$ \\
\hline & 3 & 504 & $95.87 \pm 1.36$ & $95.53 \pm 1.35$ & $93.33 \pm 1.30$ \\
\hline & 4 & 672 & $96.27 \pm 1.10$ & $96.20 \pm 1.04$ & $94.07 \pm 1.55$ \\
\hline & 5 & 840 & $96.47 \pm 1.60$ & $96.47 \pm 1.69$ & $95.13 \pm 1.26$ \\
\hline & 6 & 1008 & $96.60 \pm 1.46$ & $96.27 \pm 1.51$ & $95.27 \pm 1.87$ \\
\hline & 7 & 1176 & $95.40 \pm 1.73$ & $95.87 \pm 1.40$ & $95.07 \pm 1.55$ \\
\hline
\end{tabular}

For grayscale images, it has been observed that as the level of image decomposition increases, the classification accuracy for DWTFOSLBP ${ }^{\mathrm{u} 2}$ and DWTFOSLBP ${ }^{\mathrm{ri}}$ techniques grows up to the $4^{\text {th }}$ level of image decomposition and decreases slightly thereafter. In case of DWTFOSLBPriu2 and DWTFOSLBP-HF the increase in the classification accuracy is shown up to the $5^{\text {th }}$ and $6^{\text {th }}$ level of image decomposition, respectively, and thereafter a marginal decrement in the classification accuracy has been noticed as shown in table 4. Similar trends have been observed for classification accuracy achieved by texture features of RGB images as shown in table 5 .

4.4d Proposed DWT based hybrid texture features using feature vector data selected by mRMR technique: The classification accuracy offered by a large number of complex features produced by DWT based hybrid texture features have increased computational time of classifiers. The efficiency of the feature vector data selected by FS method in terms of classification accuracy for both grayscale and RGB images has been presented in table 6 and table 7, respectively. 
Table 5. Classification accuracy achieved using full feature vector data of DWT based hybrid texture features extracted from RGB images.

\begin{tabular}{|c|c|c|c|c|c|}
\hline \multirow[b]{2}{*}{ Technique } & \multirow[b]{2}{*}{ IDL } & \multicolumn{4}{|c|}{$\% \mathrm{CA} \pm \mathrm{SD}$ achieved by classifiers } \\
\hline & & $\overline{\mathrm{NoF}}$ & Linear SVM & RBF kernel SVM & $\mathrm{RF}$ \\
\hline \multirow[t]{7}{*}{ DWTFOSLBP ${ }^{\mathrm{u} 2}$} & 1 & 252 & $92.67 \pm 1.81$ & $91.60 \pm 1.76$ & $85.07 \pm 2.69$ \\
\hline & 2 & 504 & $96.47 \pm 1.86$ & $95.20 \pm 1.69$ & $92.40 \pm 2.23$ \\
\hline & 3 & 756 & $97.73 \pm 1.05$ & $97.00 \pm 1.64$ & $95.13 \pm 1.26$ \\
\hline & 4 & 1008 & $98.40 \pm 0.64$ & $98.13 \pm 0.88$ & $96.00 \pm 1.13$ \\
\hline & 5 & 1260 & $97.73 \pm 1.14$ & $97.67 \pm 1.41$ & $96.33 \pm 1.23$ \\
\hline & 6 & 1512 & $97.73 \pm 1.14$ & $97.40 \pm 1.27$ & $96.53 \pm 1.03$ \\
\hline & 7 & 1764 & $97.87 \pm 1.29$ & $97.73 \pm 1.00$ & $96.27 \pm 1.26$ \\
\hline \multirow[t]{7}{*}{ DWTFOSLBP ${ }^{\mathrm{ri}}$} & 1 & 160 & $88.93 \pm 1.81$ & $90.53 \pm 2.08$ & $84.73 \pm 2.71$ \\
\hline & 2 & 320 & $95.20 \pm 1.40$ & $94.60 \pm 1.82$ & $91.27 \pm 2.11$ \\
\hline & 3 & 480 & $97.60 \pm 1.23$ & $96.73 \pm 1.19$ & $95.13 \pm 1.18$ \\
\hline & 4 & 640 & $97.73 \pm 0.90$ & $97.33 \pm 0.83$ & $95.33 \pm 0.89$ \\
\hline & 5 & 800 & $97.80 \pm 0.77$ & $97.27 \pm 0.91$ & $96.00 \pm 0.89$ \\
\hline & 6 & 960 & $97.93 \pm 0.97$ & $97.20 \pm 0.98$ & $96.73 \pm 1.23$ \\
\hline & 7 & 1120 & $97.67 \pm 0.85$ & $96.80 \pm 1.43$ & $96.13 \pm 0.98$ \\
\hline \multirow[t]{7}{*}{ DWTFOSLBP ${ }^{\text {riu2 }}$} & 1 & 56 & $87.07 \pm 2.74$ & $88.67 \pm 2.92$ & $83.00 \pm 2.63$ \\
\hline & 2 & 112 & $94.33 \pm 1.61$ & $94.20 \pm 1.54$ & $89.73 \pm 1.84$ \\
\hline & 3 & 168 & $97.40 \pm 1.42$ & $96.27 \pm 1.55$ & $93.73 \pm 1.10$ \\
\hline & 4 & 224 & $98.07 \pm 1.42$ & $97.60 \pm 1.10$ & $95.87 \pm 0.98$ \\
\hline & 5 & 280 & $98.27 \pm 0.78$ & $97.87 \pm 0.98$ & $96.00 \pm 1.30$ \\
\hline & 6 & 336 & $97.87 \pm 1.12$ & $98.13 \pm 0.75$ & $96.13 \pm 0.93$ \\
\hline & 7 & 392 & $97.33 \pm 0.90$ & $97.47 \pm 1.60$ & $95.80 \pm 1.48$ \\
\hline \multirow[t]{7}{*}{ DWTFOSLBP-HF } & 1 & 168 & $87.07 \pm 3.00$ & $88.07 \pm 2.32$ & $86.13 \pm 2.01$ \\
\hline & 2 & 336 & $93.93 \pm 2.10$ & $94.20 \pm 1.55$ & $91.53 \pm 1.63$ \\
\hline & 3 & 504 & $96.20 \pm 0.90$ & $96.73 \pm 1.35$ & $95.13 \pm 1.21$ \\
\hline & 4 & 672 & $97.67 \pm 1.00$ & $97.60 \pm 1.05$ & $96.13 \pm 1.21$ \\
\hline & 5 & 840 & $97.47 \pm 0.82$ & $97.73 \pm 1.48$ & $96.53 \pm 0.69$ \\
\hline & 6 & 1008 & $97.60 \pm 1.05$ & $97.60 \pm 1.18$ & $96.33 \pm 1.05$ \\
\hline & 7 & 1176 & $97.27 \pm 1.11$ & $97.47 \pm 1.21$ & $96.60 \pm 1.19$ \\
\hline
\end{tabular}

The selected feature subset of DWTFOSLBP ${ }^{\mathrm{u} 2}$ texture features extracted from the grayscale images produced a classification accuracy of $98.60 \pm 0.66 \%$ (300 features) at the $7^{\text {th }}$ level of image decomposition compared to $97.67 \pm 0.79 \%$ using full feature dataset at the $4^{\text {th }}$ level of image decomposition by linear SVM classifier. Amongst the proposed texture feature extraction techniques DWTFOSLBP-HF has obtained the best classification accuracy of $99.00 \pm 0.79 \%$ at the $5^{\text {th }}$ level of image decomposition with linear SVM classifier. Other techniques have also shown improvement in the classification accuracy compared to full feature vector data as shown in table 6 . The classification accuracy obtained by the proposed techniques using RBF kernel SVM classifier $(98.80 \pm 0.69 \%)$ is also comparable with the accuracy achieved by linear SVM classifier. The classification accuracy produced by RF classifier $(96.20 \pm 1.51 \%)$ is not up to the mark in comparison with the other two classifiers.

The classification accuracy achieved by the classifiers for feature vector data subsets selected by mRMR method from the RGB image texture features, obtained with proposed techniques is listed in table 7 . Here, again amongst the proposed texture feature extraction techniques, the best 
Table 6. Classification accuracy achieved using mRMR based reduced feature vector data of DWT based hybrid texture features extracted from grayscale images.

\begin{tabular}{|c|c|c|c|c|c|c|c|}
\hline \multirow[b]{2}{*}{ Technique } & \multirow[b]{2}{*}{ IDL } & \multicolumn{6}{|c|}{$\% \mathrm{CA} \pm \mathrm{SD}$ achieved by classifiers } \\
\hline & & $\overline{\mathrm{NoF}}$ & Linear SVM & $\mathrm{NoF}$ & RBF kernel SVM & $\mathrm{NoF}$ & $\mathrm{RF}$ \\
\hline \multirow[t]{7}{*}{ DWTFOSLBP ${ }^{\mathrm{u} 2}$} & 1 & 150 & $91.33 \pm 2.08$ & 150 & $91.87 \pm 2.01$ & 100 & $84.60 \pm 2.85$ \\
\hline & 2 & 200 & $96.20 \pm 1.26$ & 200 & $95.80 \pm 1.29$ & 300 & $90.60 \pm 2.38$ \\
\hline & 3 & 300 & $97.73 \pm 0.72$ & 300 & $97.53 \pm 1.51$ & 300 & $93.67 \pm 1.45$ \\
\hline & 4 & 300 & $98.13 \pm 0.88$ & 300 & $98.33 \pm 0.79$ & 175 & $95.00 \pm 1.76$ \\
\hline & 5 & 275 & $98.40 \pm 0.84$ & 300 & $98.27 \pm 0.47$ & 150 & $95.47 \pm 1.21$ \\
\hline & 6 & 300 & $98.33 \pm 0.79$ & 250 & $98.33 \pm 0.96$ & 175 & $95.93 \pm 0.91$ \\
\hline & 7 & 300 & $98.60 \pm 0.66$ & 250 & $98.33 \pm 0.79$ & 175 & $95.80 \pm 1.04$ \\
\hline \multirow[t]{7}{*}{ DWTFOSLBP ${ }^{\text {ri }}$} & 1 & 155 & $85.00 \pm 1.92$ & 155 & $87.13 \pm 2.37$ & 75 & $83.27 \pm 3.05$ \\
\hline & 2 & 275 & $92.80 \pm 1.45$ & 75 & $93.80 \pm 1.96$ & 200 & $89.53 \pm 2.01$ \\
\hline & 3 & 150 & $96.87 \pm 1.37$ & 225 & $97.00 \pm 0.72$ & 175 & $93.53 \pm 1.63$ \\
\hline & 4 & 100 & $97.47 \pm 0.88$ & 175 & $97.93 \pm 0.86$ & 150 & $94.87 \pm 1.48$ \\
\hline & 5 & 200 & $98.20 \pm 1.09$ & 175 & $98.13 \pm 0.76$ & 125 & $95.93 \pm 1.57$ \\
\hline & 6 & 300 & $98.27 \pm 0.90$ & 200 & $98.20 \pm 0.63$ & 150 & $95.80 \pm 1.99$ \\
\hline & 7 & 150 & $98.33 \pm 0.79$ & 200 & $98.27 \pm 0.78$ & 150 & $95.93 \pm 1.52$ \\
\hline \multirow{7}{*}{ DWTFOSLBP ${ }^{\text {riu2 }}$} & 1 & 50 & $86.00 \pm 3.68$ & 55 & $86.93 \pm 2.29$ & 40 & $79.20 \pm 3.15$ \\
\hline & 2 & 110 & $93.13 \pm 1.63$ & 75 & $93.13 \pm 1.75$ & 100 & $86.93 \pm 1.89$ \\
\hline & 3 & 160 & $96.73 \pm 0.86$ & 100 & $96.67 \pm 0.89$ & 165 & $91.40 \pm 1.31$ \\
\hline & 4 & 200 & $97.80 \pm 0.71$ & 175 & $97.73 \pm 1.10$ & 75 & $93.80 \pm 1.69$ \\
\hline & 5 & 150 & $98.13 \pm 0.76$ & 150 & $97.80 \pm 0.55$ & 175 & $94.73 \pm 1.11$ \\
\hline & 6 & 175 & $98.13 \pm 0.82$ & 175 & $98.00 \pm 0.83$ & 300 & $94.93 \pm 1.51$ \\
\hline & 7 & 175 & $98.13 \pm 0.53$ & 175 & $98.13 \pm 0.69$ & 175 & $94.87 \pm 1.63$ \\
\hline \multirow{7}{*}{ DWT FOSLBP-HF } & 1 & 50 & $86.53 \pm 2.56$ & 100 & $86.07 \pm 3.83$ & 50 & $84.93 \pm 1.67$ \\
\hline & 2 & 100 & $94.06 \pm 1.61$ & 150 & $94.53 \pm 1.60$ & 125 & $90.33 \pm 1.73$ \\
\hline & 3 & 175 & $97.67 \pm 1.38$ & 150 & $97.53 \pm 0.63$ & 175 & $94.20 \pm 1.81$ \\
\hline & 4 & 275 & $98.53 \pm 0.47$ & 250 & $98.47 \pm 0.89$ & 175 & $95.00 \pm 1.01$ \\
\hline & 5 & 275 & $99.00 \pm 0.79$ & 225 & $98.60 \pm 0.49$ & 175 & $95.93 \pm 1.52$ \\
\hline & 6 & 275 & $98.80 \pm 0.53$ & 275 & $98.73 \pm 0.58$ & 175 & $96.20 \pm 1.51$ \\
\hline & 7 & 200 & $98.73 \pm 0.38$ & 275 & $98.80 \pm 0.69$ & 175 & $95.93 \pm 1.55$ \\
\hline
\end{tabular}

classification accuracy of $99.20 \pm 0.42 \%$ has been achieved by DWTFOSLBP-HF features at the $6^{\text {th }}$ level of image decomposition with 300 features by linear SVM classifier. When classified with RBF kernel SVM classifier, the DWTFOSLBP ${ }^{\text {ri }}$ features have resulted into a classification accuracy of $99.13 \pm 0.45 \%$ at the $6^{\text {th }}$ level of image decomposition using 225 features only. The classification accuracy obtained for all the DWT based hybrid texture feature extraction techniques with linear and RBF kernel SVM classifiers are almost comparable with each other for the given feature vector data. But the RF classifier could not produce comparable results for the same.

At the lower level of image decomposition, a considerable change in the classification accuracy has been produced by the classifiers for texture features extracted from grayscale and RGB images. But, at the higher level of image decomposition the classification accuracy achieved by the classifiers with DWT based hybrid texture features for grayscale images are comparable with those of RGB images. Thus, it is recognized that RGB to grayscale image conversion does not cause significant loss of information which would create problems in the classification of hardwood species database for the proposed texture feature extraction techniques. Though RGB 
Table 7. Classification accuracy achieved using mRMR based reduced feature vector data of DWT based hybrid texture features extracted from RGB images.

\begin{tabular}{|c|c|c|c|c|c|c|c|}
\hline \multirow[b]{2}{*}{ Technique } & \multirow[b]{2}{*}{ IDL } & \multicolumn{6}{|c|}{$\% \mathrm{CA} \pm \mathrm{SD}$ achieved by classifiers } \\
\hline & & $\overline{\mathrm{NoF}}$ & Linear SVM & NoF & RBF kernel SVM & $\mathrm{NoF}$ & $\mathrm{RF}$ \\
\hline \multirow[t]{7}{*}{ DWTFOSLBP ${ }^{\mathrm{u} 2}$} & 1 & 175 & $93.13 \pm 1.81$ & 100 & $92.80 \pm 1.69$ & 50 & $87.87 \pm 2.21$ \\
\hline & 2 & 300 & $96.93 \pm 1.64$ & 225 & $96.73 \pm 1.31$ & 125 & $93.00 \pm 1.67$ \\
\hline & 3 & 225 & $97.73 \pm 0.78$ & 225 & $97.87 \pm 0.88$ & 150 & $95.80 \pm 1.34$ \\
\hline & 4 & 225 & $98.53 \pm 0.82$ & 225 & $98.67 \pm 0.70$ & 125 & $96.47 \pm 1.14$ \\
\hline & 5 & 200 & $98.60 \pm 1.06$ & 275 & $98.87 \pm 0.55$ & 200 & $97.27 \pm 1.18$ \\
\hline & 6 & 225 & $98.80 \pm 0.82$ & 250 & $98.93 \pm 0.56$ & 175 & $96.93 \pm 1.18$ \\
\hline & 7 & 225 & $98.80 \pm 0.82$ & 250 & $98.93 \pm 0.47$ & 175 & $97.07 \pm 1.23$ \\
\hline \multirow[t]{7}{*}{ DWTFOSLBP ${ }^{\text {ri }}$} & 1 & 150 & $89.53 \pm 1.48$ & 150 & $90.73 \pm 1.42$ & 50 & $86.33 \pm 2.02$ \\
\hline & 2 & 250 & $95.27 \pm 1.29$ & 125 & $96.07 \pm 1.42$ & 200 & $92.27 \pm 1.61$ \\
\hline & 3 & 225 & $97.87 \pm 0.88$ & 200 & $98.00 \pm 1.30$ & 175 & $95.80 \pm 1.63$ \\
\hline & 4 & 175 & $98.47 \pm 0.77$ & 225 & $98.67 \pm 0.44$ & 175 & $96.73 \pm 1.06$ \\
\hline & 5 & 175 & $98.53 \pm 0.76$ & 175 & $98.87 \pm 0.45$ & 300 & $96.93 \pm 1.05$ \\
\hline & 6 & 300 & $98.80 \pm 0.76$ & 225 & $99.13 \pm 0.45$ & 150 & $97.13 \pm 1.44$ \\
\hline & 7 & 200 & $98.87 \pm 0.63$ & 250 & $98.93 \pm 0.34$ & 225 & $96.93 \pm 0.84$ \\
\hline \multirow[t]{7}{*}{ DWTFOSLBP ${ }^{\text {riu2 }}$} & 1 & 55 & $87.07 \pm 1.51$ & 50 & $89.27 \pm 2.91$ & 55 & $82.73 \pm 2.25$ \\
\hline & 2 & 100 & $94.53 \pm 1.50$ & 75 & $94.73 \pm 1.39$ & 100 & $90.20 \pm 1.96$ \\
\hline & 3 & 150 & $97.87 \pm 0.93$ & 125 & $97.06 \pm 1.26$ & 165 & $94.47 \pm 1.81$ \\
\hline & 4 & 200 & $98.20 \pm 1.26$ & 150 & $98.13 \pm 1.47$ & 150 & $95.80 \pm 1.21$ \\
\hline & 5 & 150 & $98.40 \pm 0.84$ & 175 & $98.40 \pm 0.78$ & 200 & $96.27 \pm 1.05$ \\
\hline & 6 & 125 & $98.67 \pm 0.88$ & 150 & $98.60 \pm 0.66$ & 150 & $96.33 \pm 1.34$ \\
\hline & 7 & 125 & $98.87 \pm 0.77$ & 125 & $98.60 \pm 0.73$ & 75 & $96.40 \pm 1.45$ \\
\hline \multirow[t]{7}{*}{ DWTFOSLBP-HF } & 1 & 150 & $87.33 \pm 2.72$ & 125 & $88.47 \pm 2.01$ & 100 & $87.13 \pm 2.55$ \\
\hline & 2 & 150 & $94.33 \pm 1.76$ & 150 & $94.87 \pm 1.26$ & 200 & $92.87 \pm 1.18$ \\
\hline & 3 & 200 & $98.20 \pm 1.00$ & 175 & $97.87 \pm 1.25$ & 150 & $95.33 \pm 1.66$ \\
\hline & 4 & 300 & $98.93 \pm 0.47$ & 300 & $98.47 \pm 0.77$ & 300 & $96.80 \pm 1.12$ \\
\hline & 5 & 300 & $99.07 \pm 0.47$ & 250 & $98.87 \pm 0.55$ & 300 & $97.07 \pm 1.26$ \\
\hline & 6 & 300 & $99.20 \pm 0.42$ & 275 & $98.93 \pm 0.47$ & 175 & $97.13 \pm 1.14$ \\
\hline & 7 & 300 & $99.13 \pm 0.55$ & 250 & $99.07 \pm 0.47$ & 300 & $97.33 \pm 0.94$ \\
\hline
\end{tabular}

image texture features have better discriminative features, it takes almost thrice the time taken by grayscale images to extract features from these images.

Tables 2-7 given in the results section comprise the comprehensive results obtained on the entire dataset. The classification accuracy presented in these tables is the average value of classification accuracy achieved in each case of 10-fold cross validation approach along with SD. In figures 4 and 5, the error bar plot of the results in tables 2-7 is presented to evaluate the comparative performance of different classifiers and different features (state-of-the-art and DWTFOSLBP texture features for grayscale and color images with full feature vector data and mRMR based reduced feature vector data).

Here, M1, M2, M3 and M4 denote DWTFOSLBP ${ }^{\mathrm{u} 2}$, DWTFOSLBP ${ }^{\mathrm{ri}}$, DWTFOSLBP ${ }^{\text {riu2 }}$ and DWTFOSLBP-HF techniques, respectively. It is observed that proposed techniques (M1-M4) have obtained maximum classification accuracy with minimum value of SD. Thus, the DWTFOSLBP techniques have outperformed the state-of-the-art texture feature extraction techniques for microscopic images of hardwood species. 

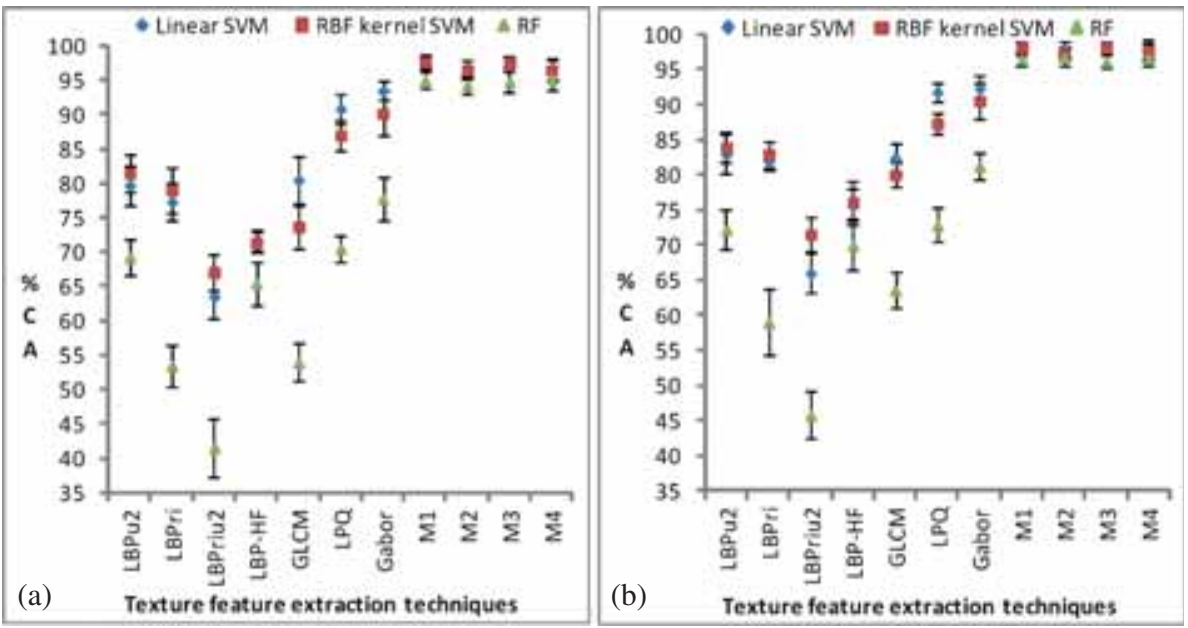

Figure 4. Error bar plot with SD for classification accuracy for full feature vector data of state-of-the-art and DWT based hybrid texture feature extraction techniques for (a) grayscale images, and (b) color images.

\subsection{Performance evaluation of texture feature extraction techniques using randomly divided database}

In section 4.4 it has been observed that the classification accuracy results obtained by mRMR based feature vector data subsets have been better than the full feature vector data. Therefore, in this section only reduced feature vector data has been considered in the investigation.

The classification accuracy achieved for the different proportions of training and testing dataset by three classifiers for state-of-the-art and DWTFOSLBP texture features of grayscale
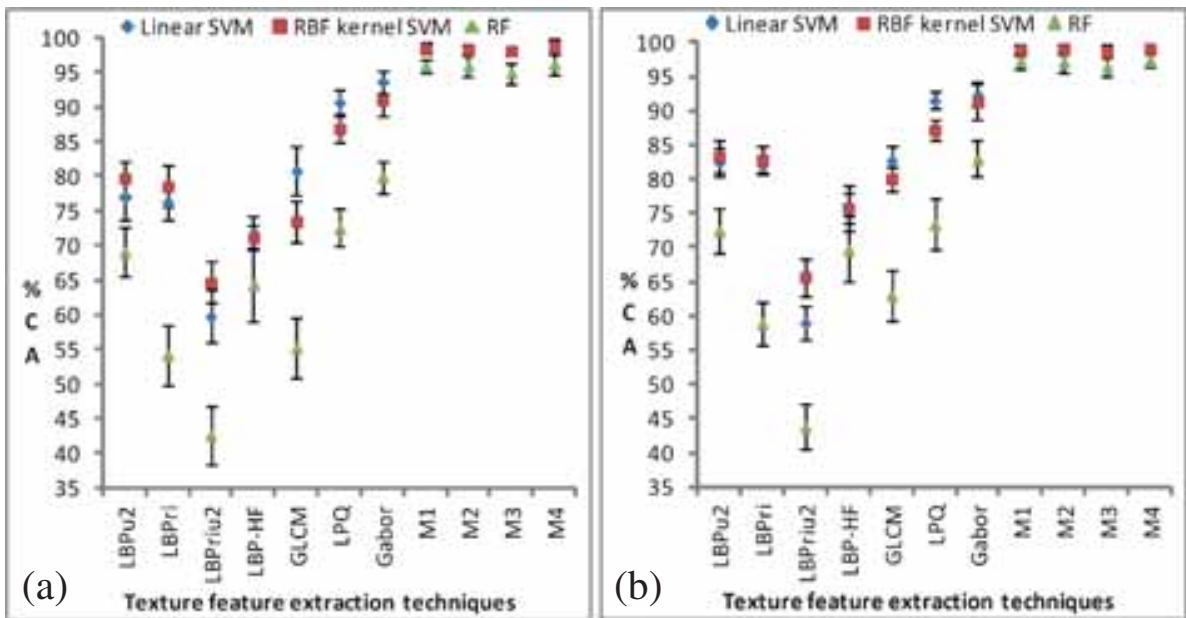

Figure 5. Error bar plot with SD for classification accuracy for the mRMR reduced feature vector data of state-of-the-art and DWT based hybrid texture feature extraction techniques for (a) grayscale images, and (b) color images. 
and RGB images are given in table 8 and table 9 , respectively. It is observed from table 8 that DWTFOSLBP techniques have acquired significant features of grayscale images of hardwood species and the best classification accuracy of $96.67 \%$ and $93.33 \%$ has been achieved for DWTFOSLBP-HF texture features with linear SVM classifiers for 80/20 and 50/50 training and testing ratios. The DWTFOSLBP ${ }^{\text {ri }}$ and DWTFOSLBP ${ }^{\text {riu2 }}$ texture features have produced best classification accuracy of $93.67 \%$ and $95.78 \%$ for $60 / 40$ and $70 / 30$ proportions of training and testing ratios with linear SVM classifier. The RBF kernel SVM classifier has also produced

Table 8. Classification accuracy achieved for randomly divided database using state-of-the-art and DWT based hybrid texture features extracted from grayscale images.

\begin{tabular}{|c|c|c|c|c|c|c|c|c|c|c|}
\hline \multirow[b]{2}{*}{ Classifier } & \multirow{2}{*}{$\begin{array}{l}\text { Texture feature } \\
\text { techniques }\end{array}$} & \multicolumn{9}{|c|}{$\%$ CA for different proportions of train and test dataset } \\
\hline & & IDL & $\overline{\mathrm{NoF}}$ & $80 / 20$ & $\mathrm{NoF}$ & $70 / 30$ & $\mathrm{NoF}$ & $60 / 40$ & $\mathrm{NoF}$ & $50 / 50$ \\
\hline \multirow[t]{12}{*}{ Linear SVM } & $\mathrm{LBP}^{\mathrm{u} 2}$ & - & 55 & 72.33 & 55 & 66.00 & 55 & 64.67 & 50 & 60.40 \\
\hline & $\mathrm{LBP}^{\mathrm{ri}}$ & - & 35 & 71.33 & 35 & 65.56 & 35 & 63.33 & 35 & 59.60 \\
\hline & LBP ${ }^{\text {riu} 2 ~}$ & - & 9 & 61.00 & 9 & 57.11 & 9 & 53.67 & 9 & 54.13 \\
\hline & LBP-HF & - & 37 & 66.67 & 37 & 57.55 & 37 & 52.33 & 37 & 50.80 \\
\hline & FOS & - & 4 & 16.67 & 4 & 16.00 & 4 & 16.00 & 4 & 14.80 \\
\hline & LPQ & - & 175 & 84.33 & 175 & 79.33 & 175 & 74.67 & 175 & 72.93 \\
\hline & GLCM & - & 70 & 76.00 & 70 & 75.33 & 70 & 69.50 & 70 & 67.60 \\
\hline & Gabor & - & 115 & 86.67 & 115 & 85.11 & 110 & 82.50 & 110 & 80.80 \\
\hline & DWTFOSLBP ${ }^{\mathrm{u} 2}$ & 7 & 300 & 95.33 & 250 & 94.44 & 225 & 92.83 & 250 & 92.67 \\
\hline & DWTFOSLBP ${ }^{\mathrm{ri}}$ & 7 & 175 & 95.67 & 275 & 95.56 & 225 & 93.67 & 100 & 92.13 \\
\hline & DWTFOSLBP riu2 & 7 & 175 & 96.33 & 200 & 95.78 & 200 & 93.33 & 175 & 92.00 \\
\hline & DWTFOSLBP-HF & 5 & 225 & 96.67 & 200 & 95.33 & 125 & 93.50 & 150 & 93.33 \\
\hline \multirow{12}{*}{ RBF kernel SVM } & $\mathrm{LBP}^{\mathrm{u} 2}$ & - & 55 & 69.33 & 55 & 66.00 & 55 & 60.33 & 55 & 56.93 \\
\hline & $\mathrm{LBP}^{\mathrm{ri}}$ & - & 35 & 71.33 & 35 & 66.89 & 35 & 64.50 & 30 & 61.07 \\
\hline & $\mathrm{LBP}^{\text {riu2 }}$ & - & 9 & 63.67 & 9 & 58.67 & 9 & 57.83 & 9 & 53.33 \\
\hline & LBP-HF & - & 37 & 65.67 & 37 & 58.00 & 37 & 53.00 & 37 & 49.73 \\
\hline & FOS & - & 4 & 37.00 & 4 & 35.11 & 4 & 33.83 & 4 & 33.07 \\
\hline & LPQ & - & 175 & 79.67 & 225 & 71.56 & 225 & 67.33 & 200 & 64.00 \\
\hline & GLCM & - & 70 & 68.00 & 70 & 64.67 & 70 & 61.67 & 70 & 60.40 \\
\hline & Gabor & - & 115 & 84.00 & 115 & 81.55 & 115 & 76.00 & 110 & 74.93 \\
\hline & DWTFOSLBP $^{\mathrm{u} 2}$ & 7 & 300 & 96.00 & 225 & 95.11 & 275 & 93.33 & 275 & 92.53 \\
\hline & DWTFOSLBP ${ }^{\text {ri }}$ & 7 & 300 & 96.67 & 300 & 95.33 & 100 & 93.00 & 275 & 91.87 \\
\hline & DWTFOSLBP riu2 & 7 & 200 & 96.00 & 200 & 94.67 & 175 & 92.83 & 225 & 91.60 \\
\hline & DWTFOSLBP-HF & 7 & 200 & 95.67 & 225 & 94.89 & 250 & 93.50 & 250 & 93.06 \\
\hline \multirow[t]{12}{*}{ RF } & $\mathrm{LBP}^{\mathrm{u} 2}$ & - & 50 & 58.00 & 55 & 52.22 & 55 & 50.67 & 50 & 48.40 \\
\hline & $\mathrm{LBP}^{\mathrm{ri}}$ & - & 35 & 48.33 & 35 & 44.89 & 35 & 41.50 & 35 & 36.80 \\
\hline & LBP riu2 & - & 9 & 34.88 & 9 & 34.50 & 9 & 34.33 & 9 & 31.73 \\
\hline & LBP-HF & - & 37 & 58.33 & 37 & 50.89 & 37 & 52.00 & 37 & 48.13 \\
\hline & FOS & - & 4 & 33.67 & 4 & 29.33 & 4 & 28.17 & 4 & 27.60 \\
\hline & LPQ & - & 150 & 65.67 & 75 & 57.11 & 225 & 54.50 & 75 & 51.07 \\
\hline & GLCM & - & 60 & 51.33 & 50 & 49.11 & 50 & 48.83 & 70 & 46.67 \\
\hline & Gabor & - & 50 & 76.67 & 50 & 73.11 & 50 & 68.31 & 75 & 67.20 \\
\hline & DWTFOSLBP ${ }^{\mathrm{u} 2}$ & 6 & 225 & 93.00 & 150 & 90.67 & 250 & 90.33 & 175 & 89.60 \\
\hline & DWTFOSLBP ${ }^{r i}$ & 7 & 250 & 93.67 & 300 & 92.67 & 150 & 91.67 & 150 & 89.73 \\
\hline & DWTFOSLBP ${ }^{\text {riu2 }}$ & 6 & 225 & 92.00 & 225 & 90.44 & 250 & 90.33 & 250 & 88.53 \\
\hline & DWTFOSLBP-HF & 6 & 200 & 92.67 & 175 & 92.00 & 100 & 91.17 & 175 & 89.87 \\
\hline
\end{tabular}


Table 9. Classification accuracy achieved for randomly divided database using state-of-the-art and DWT based hybrid texture features extracted from RGB images.

\begin{tabular}{|c|c|c|c|c|c|c|c|c|c|c|}
\hline \multirow[b]{2}{*}{ Classifier } & \multirow{2}{*}{$\begin{array}{c}\text { Texture feature } \\
\text { techniques }\end{array}$} & \multirow[b]{2}{*}{ IDL } & \multicolumn{8}{|c|}{$\%$ CA for different proportions of train and test dataset } \\
\hline & & & $\mathrm{NoF}$ & $80 / 20$ & $\mathrm{NoF}$ & $70 / 30$ & $\mathrm{NoF}$ & $60 / 40$ & $\mathrm{NoF}$ & $50 / 50$ \\
\hline \multirow[t]{12}{*}{ Linear SVM } & $\mathrm{LBP}^{\mathrm{u} 2}$ & - & 55 & 76.67 & 55 & 73.11 & 50 & 70.17 & 50 & 64.53 \\
\hline & $\mathrm{LBP}^{\mathrm{ri}}$ & - & 35 & 75.67 & 35 & 71.33 & 35 & 70.50 & 35 & 68.93 \\
\hline & LBP riu2 & - & 9 & 59.00 & 9 & 52.22 & 9 & 51.83 & 9 & 50.20 \\
\hline & LBP-HF & - & 37 & 71.67 & 37 & 64.44 & 37 & 60.83 & 37 & 54.53 \\
\hline & FOS & - & 4 & 22.33 & 4 & 21.33 & 4 & 21.00 & 4 & 20.67 \\
\hline & LPQ & - & 250 & 84.00 & 250 & 79.77 & 175 & 77.67 & 250 & 73.07 \\
\hline & GLCM & - & 60 & 79.00 & 60 & 77.33 & 70 & 74.16 & 70 & 73.20 \\
\hline & Gabor & - & 115 & 86.67 & 115 & 85.78 & 110 & 84.33 & 115 & 80.80 \\
\hline & DWTFOSLBP ${ }^{\mathrm{u} 2}$ & 7 & 250 & 96.33 & 175 & 95.78 & 250 & 93.67 & 250 & 93.33 \\
\hline & DWTFOSLBP ${ }^{r i}$ & 7 & 150 & 97.33 & 150 & 96.88 & 125 & 94.67 & 125 & 93.33 \\
\hline & DWTFOSLBP riu2 & 7 & 175 & 97.33 & 200 & 96.89 & 300 & 94.00 & 150 & 92.93 \\
\hline & DWTFOSLBP-HF & 6 & 250 & 96.67 & 275 & 96.44 & 200 & 94.50 & 275 & 93.47 \\
\hline \multirow[t]{12}{*}{ RBF kernel SVM } & $\mathrm{LBP}^{\mathrm{u} 2}$ & - & 50 & 76.00 & 55 & 68.89 & 50 & 63.63 & 50 & 61.07 \\
\hline & $\mathrm{LBP}^{\mathrm{ri}}$ & - & 35 & 74.33 & 30 & 71.11 & 35 & 70.16 & 35 & 66.13 \\
\hline & LBP ${ }^{\text {riu2 }}$ & - & 9 & 61.33 & 9 & 56.00 & 9 & 53.67 & 9 & 50.13 \\
\hline & LBP-HF & - & 37 & 69.33 & 37 & 64.44 & 37 & 59.83 & 30 & 56.00 \\
\hline & FOS & - & 4 & 47.00 & 4 & 42.89 & 4 & 42.50 & 4 & 42.00 \\
\hline & LPQ & - & 100 & 79.67 & 225 & 72.22 & 250 & 68.67 & 200 & 63.20 \\
\hline & GLCM & - & 60 & 72.33 & 70 & 68.88 & 60 & 66.66 & 70 & 64.53 \\
\hline & Gabor & - & 115 & 84.00 & 115 & 83.11 & 115 & 79.17 & 110 & 74.80 \\
\hline & DWTFOSLBP ${ }^{\mathrm{u} 2}$ & 7 & 275 & 96.33 & 275 & 96.00 & 250 & 94.00 & 275 & 93.20 \\
\hline & DWTFOSLBP $^{\mathrm{ri}}$ & 6 & 125 & 96.67 & 125 & 96.66 & 250 & 94.33 & 300 & 93.73 \\
\hline & DWTFOSLBP riu2 & 6 & 175 & 96.67 & 175 & 96.44 & 250 & 94.17 & 150 & 93.20 \\
\hline & DWTFOSLBP-HF & 7 & 275 & 97.33 & 175 & 96.89 & 300 & 94.83 & 175 & 94.80 \\
\hline \multirow[t]{12}{*}{$\mathrm{RF}$} & $\mathrm{LBP}^{\mathrm{u} 2}$ & - & 55 & 65.67 & 55 & 55.77 & 55 & 53.33 & 55 & 51.46 \\
\hline & $\mathrm{LBP}^{\mathrm{ri}}$ & - & 35 & 53.33 & 30 & 47.77 & 30 & 46.50 & 35 & 43.87 \\
\hline & LBP riu2 & - & 9 & 36.67 & 9 & 36.17 & 9 & 35.16 & 9 & 32.40 \\
\hline & LBP-HF & - & 37 & 64.00 & 30 & 57.77 & 37 & 56.17 & 37 & 55.07 \\
\hline & FOS & - & 4 & 42.33 & 4 & 34.89 & 4 & 37.00 & 4 & 36.53 \\
\hline & LPQ & - & 150 & 66.00 & 125 & 57.55 & 150 & 54.17 & 250 & 52.67 \\
\hline & GLCM & - & 70 & 57.00 & 70 & 56.22 & 70 & 55.50 & 70 & 53.86 \\
\hline & Gabor & - & 100 & 78.00 & 100 & 72.22 & 50 & 71.83 & 75 & 68.40 \\
\hline & DWTFOSLBP ${ }^{\mathrm{u} 2}$ & 5 & 150 & 95.00 & 175 & 93.78 & 250 & 93.17 & 250 & 91.33 \\
\hline & DWTFOSLBP $^{\mathrm{ri}}$ & 6 & 275 & 95.67 & 175 & 95.33 & 225 & 93.50 & 300 & 91.33 \\
\hline & DWTFOSLBP riu2 & 7 & 200 & 94.67 & 175 & 94.66 & 225 & 92.67 & 275 & 90.00 \\
\hline & DWTFOSLBP-HF & 7 & 150 & 95.67 & 125 & 95.33 & 275 & 93.16 & 275 & 91.20 \\
\hline
\end{tabular}

comparable classification accuracy, but RF classifier could not match the classification accuracy results obtained by other two classifiers as listed in table 8 .

In case of DWTFOSLBP texture features extracted from RGB image as shown in table 9, the DWTFOSLBP-HF texture features have produced $97.33 \%, 96.89 \%, 94.83 \%$ and $94.80 \%$ classification accuracy for 80/20, 70/30, 60/40 and 50/50 training and testing ratios, respectively, with RBF kernel SVM classifier. Also, the DWTFOSLBP riu2 texture features have produced the best classification accuracy of $97.33 \%$ and $96.89 \%$ for $80 / 20$ and $70 / 30$ train and test ratios, respectively, using linear SVM classifier. The classification accuracy obtained by DWTFOSLBP 
texture features is much superior to the state-of-the-art texture features which are evident from table 8 and table 9 . The above analysis suggests that DWT based hybrid texture feature extraction techniques acquire significant features of the images and are well suited for the classification of hardwood species using both the 10-fold cross validation and randomly divided database approaches.

The state-of-the-art Gabor feature yields highest classification accuracy for both grayscale and color images. But to obtain Gabor texture features, for each grayscale image 40 Gabor patterns are generated and for each RGB images 120 Gabor pattern images are generated (Haghighat et al 2013). Thus, the classification accuracy has been produced at the cost of huge computation time for features extraction. Further, Gabor method is one of the multiresolution analysis approaches which is being used in texture feature extraction.

On contrary, the FOS and variants of LBP techniques are simple yet computationally efficient features extraction techniques. Further, individually the $\mathrm{LBP}^{\mathrm{u} 2}$ (LBP variants) has achieved a classification accuracy of $79.73 \pm 2.54 \%$ and $83.33 \pm 2.45 \%$ for grayscale and color images, respectively; and the FOS has achieved a classification accuracy of $36.20 \pm 2.93 \%$ and $47.53 \pm 3.36 \%$ for grayscale and color images, respectively. For a given image, the LBP variants produce local texture descriptors while the FOS is a global texture descriptor. Thus, an effort has been made by the authors to combine these descriptors to get the significant features of images at multiresolutions without involving large increase in the number of features. This is the reason that authors have chosen combination of FOS and variant of LBP at multiresolution.

The need of image decomposition to several levels in this problem for texture feature extraction is as follows: Since, the human visual perception evaluates an image on various levels of resolution at the same time (Koenderink 1984); similarly, the multiresolution analysis capability of DWT is helpful in detecting features at a unique resolution that remains undetectable at any other resolution. The microscopic images of hardwood species consist of four key elements, namely, vessels, rays, parenchyma and fibers. These elements are of various shapes and sizes. In order to get the significant information useful in the discrimination of hardwood species images, the images are examined at various levels of resolutions. For instance, large size objects are the candidates, suitable to be analyzed at coarse view (lower) resolutions; whereas smaller objects need to be examined at higher levels of resolutions. Thus DWT is used to decompose the hardwood species images to represent it into a set of frequency channels that carry the information of the image at various levels and orientations. The transformation result provides more valuable information, and the DWT coefficients of each level are different for the same characteristics of the signal/image.

Further, the original/full feature vector data produced by most of the variants of DWTFOSLBP techniques (for grayscale and RGB images) could not improve the classification accuracy significantly beyond the $4^{\text {th }} / 5^{\text {th }}$ level of image decomposition, rather same or the lower classification accuracy have been reported as shown in tables 4 and 5 . This happens because the subimages produced by DWT decomposition beyond the $4^{\text {th }} / 5^{\text {th }}$ level of image decomposition do not encompass qualitative visible information (though statistically significant features do exist). In addition, inclusion of further levels of image decomposition gives rise to computation time without considerable improvement in the classification accuracy. Therefore, in this work the authors have considered up to 7 levels of image decomposition by DWT. An important observation which is worthy to be noted is that incorporating DWT with FOS and LBP variants has extracted distinct texture features of hardwood species image. Further, combining FOS and LBP 
features together at different levels of image decomposition improves discrimination capability of a classifier for hardwood species.

\section{Conclusions}

The proposed work intends to improve effectiveness of four variants of LBP texture features for the classification of hardwood species into 75 different categories by using DWT based hybrid texture feature extraction techniques. These techniques integrate the multiresolution capability of DWT with FOS and variants of LBP. Initially, the images have been decomposed using DWT and then texture features are extracted from these images by FOS and LBP variants. The effectiveness of the proposed techniques has been investigated on an open access database of hardwood species consisting of 1,500 microscopic images samples of 75 hardwood species using 10-fold cross validation and randomly divided database approach. The performance of the texture features obtained by the proposed techniques at seven different levels of images decomposition is evaluated in terms of classification accuracy by using linear SVM, RBF kernel SVM and RF classifiers.

The comprehensive analysis of the results produced by 10 -fold cross validation approach shows that amongst the proposed texture feature extraction techniques the DWTFOSLBP ${ }^{\mathrm{u} 2}$ has obtained best classification accuracy of $97.67 \pm 0.79 \%$ and $98.40 \pm 064 \%$ for grayscale and RGB images, respectively. These accuracies are achieved for full feature vector data obtained by DWTFOSLBP $^{\mathrm{u} 2}$ at the $4^{\text {th }}$ level of image decomposition using linear SVM classifier. Further, the reduction in feature dataset is achieved by mRMR feature selection method and it is seen that DWTFOSLBP-HF texture features using linear SVM classifier produces the best classification accuracy of $99.00 \pm 0.79 \%$ (275 features) and $99.20 \pm 0.42 \%$ (300 features) at the $5^{\text {th }}$ and $6^{\text {th }}$ level of image decomposition for grayscale and RGB images, respectively. The texture features obtained by proposed techniques classified by RBF kernel SVM classifier has also delivered comparable classification accuracy for both types of image. Furthermore, it is also observed that the classification accuracy obtained by randomly dividing the hardwood species database into fix proportions of training and testing dataset using DWTFOSLBP-HF texture features is superior amongst the proposed feature extraction techniques.

The classification accuracy of the proposed work is based on the use of Daubechies wavelet (db3) for image decomposition and mRMR method for feature selections. However, the quality of texture features using other wavelet decomposition filters for image decomposition still remains unexplored. Also, different feature selection methods can be employed to provide reduced subset of feature data.

Thus, the texture features acquired by DWTFOSLBP-HF texture feature extraction technique for hardwood species are of excellent quality and no significant information loss is reported when grayscale image is employed for the classification.

\section{Acknowledgements}

The authors would like to express their earnest gratitude to Prof. Luiz Eduardo S. Oliveira, Federal University of Parana (UFPR), Department of Informatics, for providing microscopic images of hardwood species for academic research purpose. The authors are grateful to all those researchers who have made available the MATLAB codes for texture features and classifiers. The authors would like to thank the anonymous reviewers for providing valuable suggestions for improving the work. 


\section{Nomenclature}

\begin{tabular}{|c|c|c|c|}
\hline Acronym & Full form & Acronym & Full form \\
\hline $2 \mathrm{D}$ & Two dimensional & IDL & Image decomposition \\
\hline $\mathrm{ACA}$ & Ant clustering algorithm & & level \\
\hline ANN & Artificial neural network & KDA & Kernel discriminant analysis \\
\hline $\mathrm{BP}$ & Back-propagation & K-NN & K nearest neighborhood \\
\hline \multirow[t]{2}{*}{ BGLAM } & \multirow{2}{*}{$\begin{array}{l}\text { Basic gray level aura } \\
\text { matrix }\end{array}$} & LBP & Local binary pattern \\
\hline & & $\mathrm{LBP}^{\mathrm{u} 2}$ & Uniform local binary pattern \\
\hline CA & Classification accuracy & $\mathrm{LBP}^{\mathrm{ri}}$ & Rotation invariant local \\
\hline DWT & Discrete wavelet transform & & binary pattern \\
\hline \multirow[t]{2}{*}{ DWTFOSLBP ${ }^{u} 2$} & \multirow{2}{*}{$\begin{array}{l}\text { Discrete wavelet transform } \\
\text { based first-order statistics } \\
\text { uniform local binary } \\
\text { pattern }\end{array}$} & LBP ${ }^{\text {riu2 }}$ & $\begin{array}{l}\text { Rotation invariant uniform } \\
\text { local binary pattern }\end{array}$ \\
\hline & & LBP-HF & $\begin{array}{l}\text { Local binary pattern } \\
\text { histogram Fourier features }\end{array}$ \\
\hline \multirow[t]{3}{*}{ DWTFOSLBP ${ }^{\mathrm{ri}}$} & \multirow{3}{*}{$\begin{array}{l}\text { Discrete wavelet transform } \\
\text { based first-order statistics } \\
\text { rotation invariant local } \\
\text { binary pattern }\end{array}$} & LDA & $\begin{array}{l}\text { Linear discriminant } \\
\text { analysis }\end{array}$ \\
\hline & & LPQ & Local phase quantization \\
\hline & & MLP & Multilayer perceptron \\
\hline \multirow[t]{2}{*}{ DWTFOSLBP ${ }^{\text {riu2 }}$} & \multirow{2}{*}{$\begin{array}{l}\text { Discrete wavelet transform } \\
\text { based first-order statistics } \\
\text { rotation invariant uniform } \\
\text { local binary pattern }\end{array}$} & MMI & Mask matching image \\
\hline & & $\begin{array}{l}\text { MLP-BP- } \\
\text { ANN }\end{array}$ & $\begin{array}{l}\text { Multilayer perceptron } \\
\text { back propagation } \\
\text { artificial neural network }\end{array}$ \\
\hline \multirow[t]{3}{*}{ DWTFOSLBP-HF } & \multirow{3}{*}{$\begin{array}{l}\text { Discrete wavelet transform } \\
\text { based first-order statistics } \\
\text { local binary pattern } \\
\text { histogram Fourier features }\end{array}$} & mRMR & $\begin{array}{l}\text { Minimal redundancy } \\
\text { maximal relevance }\end{array}$ \\
\hline & & $\mathrm{NN}$ & Nearest neighborhood \\
\hline & & NoF & Number of features \\
\hline FOS & First-order statistics & PCA & Principal component \\
\hline FS & Feature selection & & analysis \\
\hline \multirow[t]{2}{*}{ FVDN } & \multirow{2}{*}{$\begin{array}{l}\text { Feature vector data } \\
\text { normalization }\end{array}$} & $\mathrm{RF}$ & Random forest \\
\hline & & $\mathrm{RBF}$ & Radial basis function \\
\hline \multirow[t]{2}{*}{ GLCM } & \multirow{2}{*}{$\begin{array}{l}\text { Gray level co-occurrence } \\
\text { matrix }\end{array}$} & $\mathrm{SD}$ & Standard deviation \\
\hline & & SPPD & Statistical properties \\
\hline \multirow[t]{2}{*}{ GSVD } & \multirow{2}{*}{$\begin{array}{l}\text { Generalized singular value } \\
\text { decomposition }\end{array}$} & & of pore distribution \\
\hline & & SVM & Support vector machine \\
\hline
\end{tabular}

\section{References}

Ahmad A and Yusof R 2013 The implementation of ant clustering algorithm (ACA) in clustering and classifying the tropical wood species. In: Proceedings of IEEE International Conference on Signal Image Technology \& Internet Based Systems (SITIS), pp. 720-725

Ahonen T, Matas J, He C and Pietikäinen M 2009 Rotation invariant image description with local binary pattern histogram Fourier features. Image analysis, Springer Berlin Heidelberg, pp. 61-70

Ai F-F, Bin J, Zhang Z-M, Huang J-H, Wang J-B, Liang Y-Z, Yu L and Yang Z-Y 2014 Application of random forests to select premium quality vegetable oils by their fatty acid composition. Food Chem. 143: $472-478$ 
Alexandre L A 2010 Gender recognition: A multiscale decision fusion approach. Pattern Recognit. Lett. 31: $1422-1427$

Baas P, Wheeler E and Gasson P 1989 IAWA List of microscopy features for hardwood identification. IAWA Bull. (NS) 10: 219-332

Bond B 2002 Wood identification for hardwood and softwood species native to Tennessee. Agricultural Extension Service, University of Tennessee

Breiman L 2001 Random forests. Mach. Learn. 45: 5-32

Bremananth R, Nithya B and Saipriya R 2009 Wood species recognition using GLCM and correlation. In: Proceedings of IEEE International Conference on Advances in Recent Technologies in Communication and Computing, pp. 615-619

Cavalin P R, Kapp M N, Martins J and Oliveira L E 2013 A multiple feature vector framework for forest species recognition. In: Proceedings of 28th Annual ACM Symposium on Applied Computing, pp. 16-20

Chandrashekar G and Sahin F 2014 A survey on feature selection methods. Comput. Electr. Eng. 40(1): $16-28$

Chang C-C and Lin C -J 2011 LIBSVM: A library for support vector machines. ACM Trans. Intell. Syst. Technol. (TIST) 2(3): 27

Chowdhury S, Sing J K, Basu D K and Nasipuri M 2011 Face recognition by generalized two-dimensional FLD method and multi-class support vector machines. Appl. Soft Comput. 11: 4282-4292

Chu W -S, Huang C -R and Chen C -S 2013 Gender classification from unaligned facial images using support subspaces. Inf. Sci. 221: 98-109

Cortes C and Vapnik V 1995 Support-vector networks. Mach. Learn. 20: 273-297

Coussement K and Van den Poel D 2008 Churn prediction in subscription services: An application of support vector machines while comparing two parameter-selection techniques. Expert Syst. Appl. 34(1): 313-327

Crammer K and Singer Y 2002 On the learnability and design of output codes for multiclass problems. Mach. Learn. 47(2-3): 201-233

DiFranco M D, O'Hurley G, Kay E W, Watson R W G and Cunningham P 2011 Ensemble based system for whole-slide prostate cancer probability mapping using color texture features. Comput. Med. Imaging Graph 35(7): 629-645

Doshi N P and Schaefer G 2012 Rotation-invariant local binary pattern texture classification. In: Proceedings of IEEE ELMAR, pp. 71-74

Fan R E, Chang K W, Hsieh C J, Wang X R and Lin C J 2008 LIBLINEAR: A library for large linear classification. J. Mach. Learn. Res. 9: 1871-1874

Geisser S 1993 Predictive inference. CRC press

Gonzalez R C and Woods R E 2011 Digital image processing. 3rd ed., Prentice hall

Haghighat M, Zonouz S and Abdel-Mottaleb M 2013 Identification using encrypted biometrics. Computer analysis of images and patterns, Springer, pp. 440-448

Haralick R M, Shanmugam K and Dinstein I H 1973 Textural features for image classification. IEEE Trans. Syst. Man Cybern. 3(6): 610-621

Heikkilä M, Pietikäinen M and Schmid C 2009 Description of interest regions with local binary patterns. Pattern Recognit. 42(3): 425-436

Hermanson J C and Wiedenhoeft A C 2011 A brief review of machine vision in the context of automated wood identification systems. IAWA J. 32(2): 233-250

Huang D, Shan C, Ardabilian M, Wang Y and Chen L 2011 Local binary patterns and its application to facial image analysis: a survey. IEEE Trans. Syst. Man Cybern. Part C Appl. Rev. 41(6): 765-781

Jadhav D V and Holambe R S 2009 Feature extraction using Radon and wavelet transforms with application to face recognition. Neurocomputing 72(7): 1951-1959

Jaiantilal A 2010 randomforest-matlab v0.02 http://code.google.com/p/randomforest-matlab/

Kanan C and Cottrell G W 2012 Color-to-grayscale: Does the method matter in image recognition? PLoS One 7(1): 1e297401-7e29740 
Khairuddin A S M, Khalid M and Yusof R 2011 Using two stage classification for improved Tropical wood species recognition system. In: Proceedings of 4th International Conference on Intelligent Interactive Multimedia Systems and Services (IIMSS), pp. 305-314

Khalid M, Lee E L Y, Yusof R and Nadaraj M 2008 Design of an intelligent wood species recognition system. Int. J. Simul. Syst. Sci. Technol. 9(3): 9-19

Koenderink J J 1984 The structure of images. Biol. Cybern. 50(5): 363-370

Liu M, Wang M, Wang J and Li D 2013 Comparison of random forest, support vector machine and back propagation neural network for electronic tongue data classification: Application to the recognition of orange beverage and Chinese vinegar. Sens. Actuators, B: Chem. 177: 970-980

Livens S, Scheunders P, Van de Wouwer G and Van Dyck D 1997 Wavelets for texture analysis, an overview. In: Proceedings of IET Sixth International Conference on Image Processing and Its Applications pp. 581-585

Mäenpää T 2003 The local binary pattern approach to texture analysis: Extenxions and applications. Electronic thesis PhD dissertation, Acta Univ Oul C 187, http://herkules.oulu.fi/isbn9514270762/

Mallat S G 1989 A theory for multiresolution signal decomposition: The wavelet representation. IEEE Trans. Pattern Anal. Mach. Intell. 11(7): 674-693

Martins J, Oliveira L, Nisgoski S and Sabourin R 2013 A database for automatic classification of forest species. Mach. Vision Appl. 24: 567-578

Nanni L, Lumini A and Brahnam S 2012 Survey on LBP based texture descriptors for image classification. Expert Syst. Appl. 39(3): 3634-3641

Nasirzadeh M, Khazael A A and Khalid M 2010 Woods recognition system based on local binary pattern. In: Proceedings of IEEE Second International Conference on Computational Intelligence, Communication Systems and Networks, pp. 308-313

Nikam S B and Agarwal S 2008 Wavelet energy signature and GLCM features-based fingerprint antispoofing. In: Proceedings of IEEE Second International Conference on Wavelet Analysis and Pattern Recognition, pp. 717-723

Ojala T, Pietikainen M and Harwood D 1994 Performance evaluation of texture measures with classification based on Kullback discrimination of distributions. In: Proceedings of IEEE 12th IAPR International Conference on Computer Vision \& Image Processing, pp. 582-585

Ojala T, Pietikäinen M and Harwood D 1996 A comparative study of texture measures with classification based on featured distributions. Pattern Recognit. 29(1): 51-59

Ojala T, Pietikainen M and Maenpaa T 2002 Multiresolution gray-scale and rotation invariant texture classification with local binary patterns. IEEE Trans. Pattern Anal. Mach. Intell. 24(7): 971-987

Ojansivu V and Heikkilä J 2008 Blur insensitive texture classification using local phase quantization. Image and signal processing, Springer, pp. 236-243.

Pan S and Kudo M 2012 Recognition of wood porosity based on direction insensitive feature sets. Trans. Mach. Learn. Data Mining 5: 45-62

Paula-Filho P L, Oliveira L S, Nisgoski S and Britto Jr A S 2014 Forest species recognition using macroscopic images. Mach. Vision Appl. 25(4): 1019-1031

Peng H, Long F and Ding C 2005 Feature selection based on mutual information criteria of maxdependency, max-relevance, and min-redundancy. IEEE Trans. Pattern Anal. Mach. Intell. 27(8): 12261238

Pietikäinen M, Ojala T and Xu Z 2000 Rotation-invariant texture classification using feature distributions. Pattern Recognit. 33(1): 43-52

Pietikäinen M, Hadid A, Zhao G and Ahonen T 2011 Computer vision using local binary patterns. Springer Publisher

Platt J C, Cristianini N and Shawe-Taylor J 1999 Large margin DAGs for multiclass classification. NIPS 12: $547-553$

Prasad A M, Iverson L R and Liaw A 2006 Newer classification and regression tree techniques: Bagging and random forests for ecological prediction. Ecosystems 9(2): 181-199

Saeys Y, Inza I and Larrañaga P 2007 A review of feature selection techniques in bioinformatics. Bioinformatics 23(19): 2507-2517 
Shan C, Gong S and McOwan P W 2009 Facial expression recognition based on local binary patterns: A comprehensive study. Image Vision Comput. 27: 803-816

Sripath D 2003 Efficient implementations of discrete wavelet transforms using FPGAs. Electronic thesis, Master of Science, The Florida State University http://diginole.lib.fsu.edu/etd/1599

Soh L K and Tsatsoulis C 1999 Texture analysis of SAR sea ice imagery using gray level co-occurrence matrices. IEEE Trans. Geosci. Remote Sens. 37(2): 780-795

Tou J Y, Lau P Y and Tay Y H 2007 Computer vision-based wood recognition system. In: Proceedings of International Workshop on Advanced Image Technology

Tou J Y, Tay Y H and Lau P Y 2009 Rotational invariant wood species recognition through wood species verification. In: Proceedings of IEEE International Conference on Intelligent Information and Database Systems, pp. 115-120

Uppuluri A 2010 GLCM_Features4 http://in.mathworks.com/matlabcentral/fileexchange/22354/

Wang J Z, Wiederhold G, Firschein O and Wei S X 1998 Content-based image indexing and searching using Daubechies' wavelets. Int. J. Digital Libraries 1: 311-328

Wang B H, Wang H J and Qi H N 2010 Wood recognition based on grey-level co-occurrence matrix. In: Proceedings of IEEE International Conference on Computer Application and System Modeling, pp. V1-269-272

Wang H J, Qi H N and Wang X F 2012 A new wood recognition method based on gabor entropy. Advanced intelligent computing theories and applications with aspects of artificial intelligence, Springer, pp. 435440

Wang H J, Zhang G Q and Qi H N 2013 Wood recognition using image texture features. PloS one 8: e76101

Wheeler E A and Baas P 1998 Wood identification: A review. IAWA Jl. (NS) 19: 241-264

Yadav A R, Dewal M, Anand R and Gupta S 2013 Classification of hardwood species using ANN classifier. In: Proceedings of IEEE International Conference on Computer Vision, Pattern Recognition, Image Processing and Graphics, pp. 1-5

Yadav A R, Anand R, Dewal M and Gupta S 2014 Analysis and classification of hardwood species based on Coiflet DWT feature extraction and WEKA workbench. In: Proceedings of IEEE International Conference on Signal Processing and Integrated Networks, pp. 9-13

Yaghouby F and Ayatollahi A 2010 An arrhythmia classification method based on selected features of heart rate variability signal and support vector machine-based classifier. World Congress on Medical Physics and Biomedical Engineering, Munich, Germany, Springer, pp. 1928-1931

You M and Cai C 2009 Wood classification based on PCA, 2DPCA, (2D) ${ }^{2}$ PCA and LDA. In: Proceedings of IEEE Second International Symposium on Knowledge Acquisition and Modeling, pp. 371-374

Yuan G -X, Ho C -H and Lin C -J 2012 Recent advances of large-scale linear classification. In: Proceedings of IEEE 100(9): 2584-2603

Yusof R, Rosli N R and Khalid M 2010 Using Gabor filters as image multiplier for tropical wood species recognition system. In: Proceedings of IEEE 12th International Conference on Computer Modelling and Simulation, pp. 289-294

Yusof R, Khalid M and Khairuddin A S M 2013a Application of kernel-genetic algorithm as nonlinear feature selection in tropical wood species recognition system. Comput. Electron. Agric. 93: 68-77

Yusof R, Khalid M and Khairuddin A S M 2013b Fuzzy logic-based pre-classifier for tropical wood species recognition system. Mach. Vision Appl. 24: 1589-1604

Zhao G, Ahonen T, Matas J and Pietikainen M 2012 Rotation-invariant image and video description with local binary pattern features. IEEE Trans. Image Process 21(4): 1465-1477 